\title{
Hongos liquenizados y liquenícolas de la Sierra de Albarracín (Teruel, España)
}

\author{
Estela Seriñá ${ }^{1 \star}$, Rosario Arroyo ${ }^{1}$, Elena Araujo ${ }^{1}$, Ana Rosa Burgaz ${ }^{1}$, Violeta Atienza ${ }^{2}$, \\ Samantha Fernández-Brime ${ }^{3}$, Víctor J. Rico $0^{4}$, Xavier Llimona ${ }^{3}$, Esteve Llop ${ }^{3}$, Eugenia López de Silanes ${ }^{5}$, \\ Bernarda Marcos-Laso ${ }^{6}$, Joana Marques $^{7}$, Sonia Merinero ${ }^{8}$, Sergio Pérez-Ortega ${ }^{9}$, \\ Marta Rubio-Salcedo ${ }^{8}$ y Mercedes Vivas ${ }^{4}$
}

Resumen: Seriñá, E.; Arroyo, M. R.; Araujo, E.; Burgaz, A. R.; Atienza, V.; Fernández-Brime, S.; Rico, V. J.; Llimona, X.; Llop, E.; López de Silanes, M. E.; Marcos-Laso, B.; Marques, J.; Merinero, S.; Pérez-Ortega, S.; Rubio-Salcedo, M. \& Vivas, M. 2014. Hongos liquenizados y liquenícolas de la Sierra de Albarracín (Teruel, España). Bot. Complut. 38: 35-52.

Se presenta un catálogo de 462 hongos liquenizados y liquenícolas de la Sierra de Albarracín (Teruel, Aragón, España), como resultado de la IV Campaña de Recolección organizada por la Sociedad Española de Liquenología (SEL). Diplotomma hedinii es novedad para la Península Ibérica y Lepraria leuckertiana constituye una segunda cita peninsular, 69 taxones son novedad para Aragón y 86 lo son para la provincia de Teruel.

Palabras clave: flora, líquenes, hongos liquenícolas, Lepraria, Diplotomma, Sierra de Albarracín, Teruel, Aragón, España.

Abstract: Seriñá, E.; Arroyo, M. R.; Araujo, E.; Burgaz, A. R.; Atienza, V.; Fernández-Brime, S.; Rico, V. J.; Llimona, X.; Llop, E.; López de Silanes, M. E.; Marcos-Laso, B.; Marques, J.; Merinero, S.; Pérez-Ortega, S.; Rubio-Salcedo, M. \& Vivas, M. 2014. Lichenized and lichenicolous fungi from the Sierra de Albarracín (Teruel, Spain). Bot. Complut. 38: 35-52.

A catalogue of 462 lichenized and lichenicolous fungi from Sierra de Albarracín, (Teruel, Spain), is presented as the result of the SEL (Sociedad Española de Liquenología) IV collecting field trip. Diplotomma hedinii is recorded for the first time for the Iberian Peninsula and Lepraria leuckertiana is registered for the second time. The checklist also includes 69 taxa new for Aragón Community and 86 new for the province of Teruel.

Key words: flora, lichens, lichenicolous fungi, Lepraria, Diplotomma, Sierra de Albarracín, Teruel, Aragón, Spain.

\section{INTRODUCCIÓN}

La Sierra de Albarracín, situada en el suroeste de la provincia de Teruel (Aragón), es uno de los conjuntos más sorprendentes del Sistema Ibérico tanto por sus paisajes como por su flora y su fauna. El conocimiento que existe sobre la flora de líquenes epifíticos en la provincia de Teruel no es completo, aunque si lo comparamos con otras provincias peninsulares puede considerarse bastante avanzado. Hasta principios del siglo XX las primeras aportaciones a la flora provincial fueron realizadas por Asso (1779), Colmeiro (1867a, b; 1889) y Loscos (1876-1877). Posteriormente Lázaro Ibiza (1898), Navás (1901, 1910), Pardo Sastrón $(1901,1903)$ y Pau (1903) efectúan nuevas aportaciones a la flora provincial. Más recientemente, hay que destacar, también para la provincia de Teruel,

\footnotetext{
${ }^{1}$ Departamento de Biología Vegetal I, Facultad de Biología, Universidad Complutense, 28040-Madrid, España.

${ }^{2}$ Departament de Botànica, Facultat de Ciències Biològiques, Universitat de València, 46171-Burjassot, València, España.

${ }^{3}$ Departament de Biologia Vegetal (Botànica) Facultat de Biologia, Universitat de Barcelona, Av. Diagonal 645, 08028-Barcelona, España.

${ }^{4}$ Departamento de Biología Vegetal II, Facultad de Farmacia, Universidad Complutense, 28040-Madrid, España.

${ }^{5}$ Departamento de Ingeniería de los Recursos Naturales y Medio Ambiente, E. Forestal, Universidade de Vigo, A Xunqueira s/n, 36005-Pontevedra, España.

${ }^{6}$ Departamento de Botánica. Facultad de Farmacia, Campus Universitario Miguel de Unamuno, 37007-Salamanca, España.

${ }^{7}$ Centro de Investigação em Biodiversidade e Recursos Genéticos, Universidade do Porto, Portugal.

${ }^{8}$ Área de Biodiversidad y Conservación, Universidad Rey Juan Carlos, 28933-Móstoles, Madrid, España.

${ }^{9}$ Instituto de Recursos Naturales, Centro de Ciencias Medioambientales CSIC, C/ Serrano 115-Bis, 28006-Madrid, España.

* autor para correspondencia: serina@bio.ucm.es

Recibido: 10 febrero 2014. Aceptado: 27 febrero 2014.
} 
los trabajos referidos a: Desierto de Calanda (Crespo et al. 1980), $S^{\text {a }}$ de Javalambre (Atienza et al. 1992, Fos 20002002), Sa del Tremedal (Aragón et al. 1999) y $S^{a}$ de Gúdar (Martínez et al. 2003). Burgaz et al. (2003-2005) y Calatayud et al. $(1995,1997,2001)$ aportan datos sobre citas de hongos liquenícolas y descripciones de nuevas especies en Teruel. En el ámbito de la Comunidad de Aragón señalamos a Segarra \& Catalán (2001) en cuyo trabajo, aunque inédito, realizan una completa recopilación de citas bibliográficas. Finalmente Etayo (2010) presenta un catálogo comentado con descripciones de especies nuevas y numerosas citas de líquenes y hongos liquenícolas de Aragón.

El relieve de la zona es accidentado, existiendo numerosas elevaciones con más de $1800 \mathrm{~m}$ y amplios espacios por encima de $1500 \mathrm{~m}$. Las cotas más bajas corresponden a los valles de los ríos de la cuenca del Guadalaviar. El clima es mediterráneo continental, con temperatura media anual de $8.7^{\circ} \mathrm{C}$ y oscilación térmica de $19^{\circ} \mathrm{C}$. Geológicamente, los substratos son mayoritariamente calcáreos aunque existen afloramientos silíceos de gran extensión, que en altitudes medias suelen presentarse a través de las areniscas rojas triásicas (rodenos) y en las partes más elevadas en forma de cuarcitas paleozoicas. En las áreas centrales y valle del río Guadalaviar no son raros afloramientos de terrenos arcillosos o margosos, ocasionalmente yesíferos. La Sierra de Albarracín se halla enclavada, desde el punto de vista corológico, dentro de la provincia Castellano-Maestrazgo-Manchega, en el sector celtibérico-alcarreño aunque con un notable carácter de tránsito hacia el sector maestracense (Rivas Martínez 1987). La diversidad litológica del substrato, unida al carácter montañoso de la región, provoca una multiplicidad de medios ecológicos que contribuye a aumentar la complejidad de la vegetación de la Sierra. Sobre los substratos calcáreos, la vegetación en el piso supramediterráneo son los quejigares (Quercus faginea) en situaciones protegidas y con suelos profundos, y los encinares (Quercus ilex subsp. ballota), sabinares (Juniperus thurifera) y pinares (Pinus nigra, P. pinaster, P. sylvestris) en las zonas más expuestas. En el piso oromediterráneo la vegetación corresponde a formaciones de Pino-Juniperion sabinae, pinares de Pinus sylvestris y sabinares de Juniperus sabina. Sobre los substratos ácidos de este territorio en el piso supramediterráneo, son destacables las formaciones que se desarrollan en los rodenos: pinares (Pinus pinaster) en las zonas más secas y melojares (Quercus pyrenaica) en las más húmedas. Sobre las cuarcitas del piso oromediterráneo destacan los melojares y los pinares de Pinus sylvestris (Barrera 1985, Mateo Sanz 2009).
El objetivo del presente trabajo es elaborar un catálogo de hongos liquenizados y liquenícolas de la Sierra de Albarracín, dada su gran heterogeneidad de substratos, para ampliar el conocimiento que existe sobre la flora de líquenes en la provincia de Teruel.

\section{MATERIALES Y MÉTODOS}

En el marco de la IV Campaña de Recolección (2-6 sept. 2010) organizada por la Sociedad Española de Liquenología (SEL), se realizaron muestreos en 14 localidades diferentes de la Sierra de Albarracín (Fig. 1) procurando abarcar todos los substratos y formaciones vegetales existentes. Los ejemplares fueron estudiados siguiendo la metodología clásica en liquenología y depositados en los siguientes herbarios: BCN-Lich, MA-Lich, MACB, MAF-Lich, PO, SALA-L, SANT-Lich, SPO, VAL-Lich.

El catálogo florístico se ha ordenado alfabéticamente siguiendo la nomenclatura de http://liquens.bio.ub.es/checklist/index.htm, www.indexfungorum.org y Etayo (2010). Recientes estudios moleculares han modificado sustancialmente la nomenclatura de algunos géneros tales como Aspicilia, Caloplaca, Melanelia, Phaeosporobolus, Verrucaria y Xanthoria (Arup et al. 2013, Divakar et al. 2012, Ertz et al. 2013, Gueidan et al. 2007, Sohrabi et al. 2013a, b). Hemos considerado estas actualizaciones, pero mantenemos entre paréntesis la combinación anterior. Cetraria steppae es considerada sinónimo de C. aculeata (Nadyeina et al. 2013).

Para cada taxón se proporcionan los siguientes datos: localidad (Lista 1), abreviatura del nombre del recolector (Lista 2) y substrato sobre el que ha sido recolectado (Lista 3). El símbolo $\neq$ delante del nombre, indica que se trata de una especie liquenícola. Los taxones marcados con $\left({ }^{*}\right)$ son nuevas citas para la provincia de Teruel, los marcados con $\left({ }^{* *}\right)$ para Aragón y los que tienen $\left({ }^{* *}\right)$ representan nuevas citas para la Península Ibérica.

\section{Lista 1. Localidades de muestreo}

1. Albarracín, hacia Bezas, dirección al Centro de Interpretación Dornaque, 30TXK331747, matorral sobre yesos rojos del Keuper, $1167 \mathrm{~m}$.

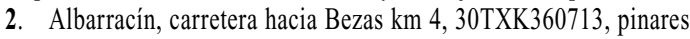
de Pinus pinaster sobre rodenos, $1393 \mathrm{~m}$.

3. Albarracín, carretera hacia Bezas km 7, cruce con pista a Gea de Albarracín, pinares de Pinus pinaster sobre rodenos, 30TXK370706, $1350 \mathrm{~m}$.

4. Bezas, parking a $2 \mathrm{~km}$ antes de llegar a Bezas, ruta $\mathrm{S} 5$ hacia pinturas rupestres de Las Tajadas, 30TXK406659, pinares de Pinus pinaster sobre rodenos, $1260 \mathrm{~m}$.

5. Albarracín, hacia Gea de Albarracín, puente de los Tres Ojos, 30TXK337771, sabinares de Juniperus thurifera, calizas verticales, $1110 \mathrm{~m}$.

6. Torres de Albarracín, carretera A1512, km 37,5, pista forestal Camino Natural del Tajo, 30TXK270753, sabinar de Juniperus thurifera, calizas verticales, $1250 \mathrm{~m}$.

7. Noguera de Albarracín, carretera A1512 hacia Orihuela del Tremedal, antes de la Peña del Castillo, pista ruta lagunas, Peñas Agudas, 30TXK163815, Pinus sylvestris y Quercus pyrenaica, pitón de cuarcitas, $1590 \mathrm{~m}$. 

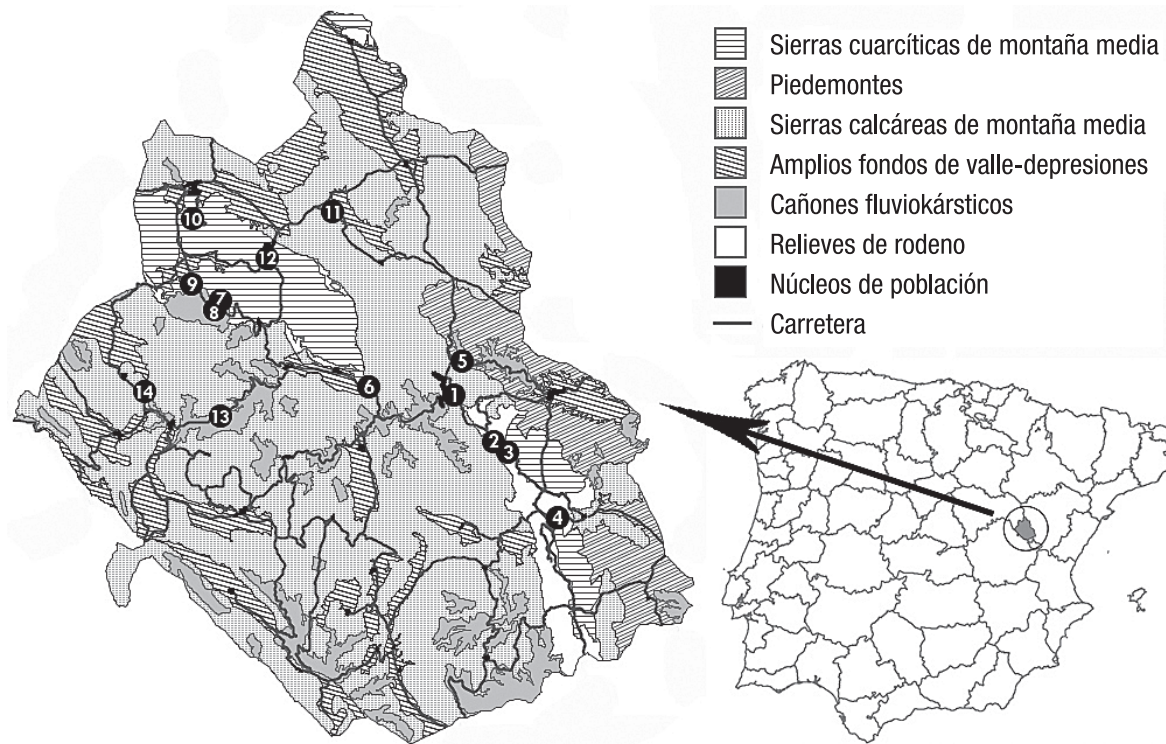

Fig. 1- Unidades de paisaje de la Sierra de Albarracín y localidades de muestreo (lista 1). Situación de la Comarca de la Sierra de Albarracín en la Península Ibérica.

8. Noguera de Albarracín hacia Orihuela del Tremedal, carretera A1512, área recreativa Peña del Castillo, 30TXK159808, Pinus sylvestris y Quercus pyrenaica, pitón diacítico, $1580 \mathrm{~m}$.

9. Puerto de Orihuela del Tremedal, 30TXK142827, Pinus sylvestris, cuarcitas, $1650 \mathrm{~m}$.

10. Orihuela del Tremedal, Santuario Ntra. Sra. del Tremedal, 30TXK143874, Pinus sylvestris, cuarcitas, $1725 \mathrm{~m}$.

11. Bronchales, carretera A2515, km 30, hacia Teruel, cerca del roble viejo, 30TXK244879, Quercus ilex subsp. ballota, Q. faginea y Juniperus thurifera, calizas, $1375 \mathrm{~m}$.

12. Bronchales, hacia Noguera, 30TXK197845, cuarcitas, $1606 \mathrm{~m}$. 13. Tramacastilla-Villar del Cobo, 30TXK163732, quejigares de Quercus faginea, $1460 \mathrm{~m}$.

14. Villar del Cobo, Casas de Búcar, cruce Guadalaviar-Griegos, pista dirección Griegos, 30TXK109749, sabinar rastrero de Juniperus sabina, calizas fosiliferas verticales, dolinas, $1425 \mathrm{~m}$.

\section{Lista 2. Nombre de los recolectores}

ARB: Ana Rosa Burgaz

BM: Bernarda Marcos-Laso

EA: Elena Araujo

ELL: Esteve Llop

ELS: Eugenia López de Silanes

ES: Estela Seriñá

JM: Joana Marques

MR: Marta Rubio-Salcedo

MV: Mercedes Vivas

RA: Rosario Arroyo

SFB: Samantha Fernández-Brime

SM: Sonia Merinero

SP0: Sergio Pérez-Ortega

VA: Violeta Atienza

VJR: Víctor J. Rico

XLL: Xavier Llimona

\section{Lista 3. Substratos}

Am: Acer monspessulanum

Bh: Berberis hispanica

Ba: base árboles

Br: briófitos

Cl: Cladonia sp.

$\mathrm{Cm}$ : Crataegus monogyna

Gh: Genista hispanica

Gs: Genista scorpius

Jc: Juniperus communis

Js: Juniperus sabina

Jt: Juniperus thurifera

L: liquenícola

Mm: madera muerta

Mu: musgos

Ot: Ononis tridentata

Pd: Populus deltoides

Pp: Pinus pinaster

Ps: Pinus sylvestris

Psp: Prunus sp.

Qc: Quercus coccifera

Qf: Quercus faginea

Qi: Quercus ilex subsp. ballota

Qp: Quercus pyrenaica

Rec: roca cuarcítica

Rcz: roca caliza

Rrd: rodenos

Sa: Salix alba

Smu: saxícola-muscícola

Ssp: Santolina sp.

Sue: terrícola

Tsp: Thymus sp.

Ys: yesos 


\section{RESULTADOS}

Se presenta un catálogo de 462 taxones repartidos en 149 géneros, de los cuales el 11,47\% son liquenícolas (representados con el símbolo $\neq$ ). Diplotomma hedinii es novedad para la Península Ibérica. Esta especie está citada para África, Asia, Europa, Norte y Centro-América (Karagöz et al. 2011) y ha sido ampliamente mencionada en la Península Ibérica por numerosos autores como D. epipolium (Ach.) Arnold o D. venustum (Körb) Körb. Lepraria leuckertiana constituye una segunda cita peninsular, fue citada por primera vez por Zedda (2000) para España, Italia, Grecia y Marruecos. Un total de 69 taxones son novedad para Aragón y 86 lo son para la provincia de Teruel. Los géneros mejor representados son Lecanora (30), Cladonia (28), Caloplaca (15), Umbilicaria (14) y Pertusaria (14).

El biotipo mayoritario es el crustáceo (44,37\%), seguido del foliáceo $(23,16 \%)$. Los biotipos que mayor número de nuevas citas aportan para la provincia de Teruel son el crustáceo (76) y el foliáceo (29) (Fig. 2). Los líquenes crustáceos son los dominantes, representando un 44\% del total (Fig. 3). Algunos de los géneros mejor representados presentan este biotipo, como Caloplaca (15), Lecanora (30) y Pertusaria (14). Los líquenes foliáceos constituyen un $23 \%$ del total, y de entre ellos destacan por su abundancia en especies los géneros Umbilicaria (14), Physcia (10) y Collema (11). Entre las especies fruticulosas (casi un 8\%), los géneros más abundantes son Ramalina (12) y Usnea (8). El biotipo dimórfico constituye un $6 \%$ del total y está representado únicamente por el género Cladonia (28). Hay que destacar el elevado número de taxones liquenícolas (53) citados en este trabajo, lo que constituye aproximadamente un $11,5 \%$ del total.

Las localidades que tienen un mayor número de taxones, en general, se corresponden con aquellas que presentan una mayor diversidad de substratos, como ocurre con las localidades 8 y 10 (Fig. 4).

\section{Acarospora bullata Anzi - 8: JM (PO8820L), Rcc.}

Acarospora cervina (Ach.) A. Massal. - 5: ARB (MACB 101365), Rcz; 6: VA (VAL-Lich 27496), Rcz; 11: BM (SALA-L 4955), MV \& VJR (MAF-Lich 16843), VA (VAL-Lich 27541), Rcz; 14: BM (SALA-L 4836), ELS (SANT 11855), JM (P08821L), SPO (SPO1512), VA (VAL-Lich 27603), Rcz.

**Acarospora flavisparsa V.J. Rico \& Candan - 7: MV \& VJR (MAFLich 16790), Rcc.

*Acarospora fuscata (Nyl.) Arnold - 10: BM (SALA-L 4981), Rcc.

**Acrocordia gemmata (Ach.) A. Massal. - 8: ELL (BCN-Lich 17511), Qp.

**Agonimia opuntiella (Buschardt \& Poelt) Vězda - 6: ELL (BCNLich 17438), Jt.

Agonimia tristicula (Nyl.) Zahlbr. -13: ELL (BCN-Lich 17663, 17666) Qf.

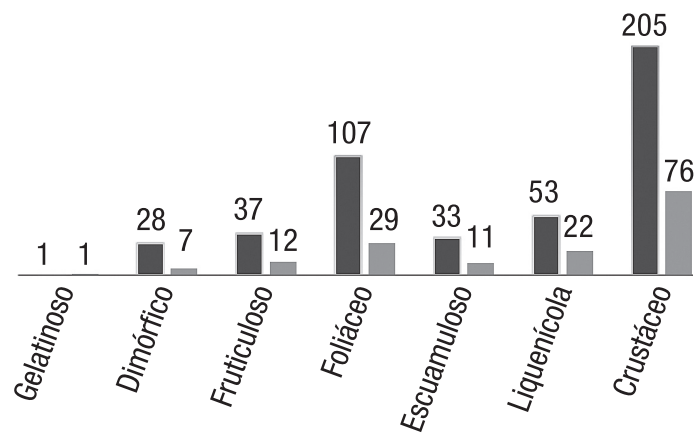

Fig. 2- Total de taxones y nuevas citas para la provincia de Teruel según el biotipo.

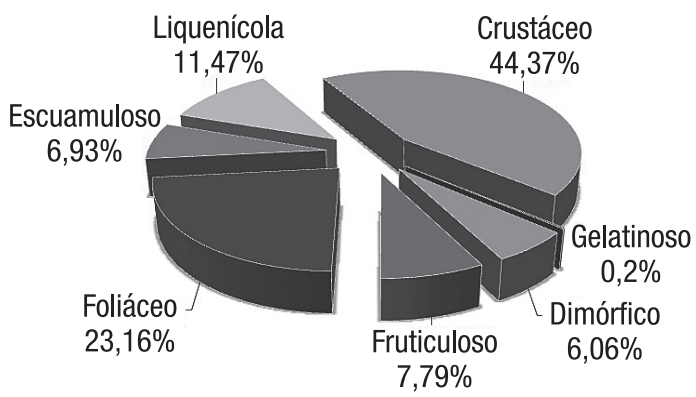

Fig. 3- Porcentaje de taxones según el biotipo en la Sierra de Albarracín.

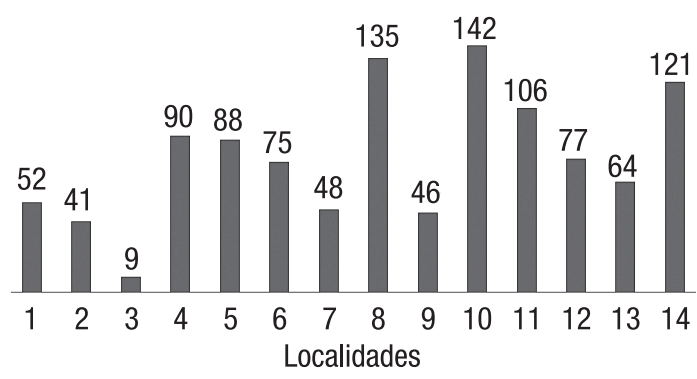

Fig. 4- Número de taxones por localidad.

Amandinea punctata (Hoffm.) Coppins \& Scheid. - 8: ELL (BCNLich 17457), Ps.

Anaptychia ciliaris (L.) Körb. - 2: ELL (BCN-Lich 17372), SFB \& XLL (BCN-Lich 17713), Rrd; 4: ARB (MACB 101366), EA, ES \& RA (MACB 102031), ELL (BCN-Lich 17384, 17389, 17393), JM (P08822L), VA (VAL-Lich 27468), Pp, Qi, Qp, Rrd, Smu; 6: ELL (BCN-Lich 17435), Jt; 7: MV \& VJR (MAF-Lich 16785, 16806), SPO (SPO-1532), Rcc, Smu; 8: EA, ES \& RA (MACB 102245, 102246, 102248, 102249, 102250, 102258), ELL (BCNLich 17469, 17480, 17483, 17492, 17509, 17531), ELS (SANT 11604), BM (SALA-L 4613), Am, Cm, Jc, Psp, Qp, Sue; 11: BM (SALA-L 4771), EA, ES \& RA (MACB 102053), ELS (SANT 
11604), ELL (BCN-Lich 17625, 17630, 17633), MR (MA-Lich 16414), VA (VAL-Lich 27542), Qf, Qi; 12: SM (MA-Lich 16369), VA (VAL-Lich 27561) Qi, Qp; 13: ARB (MACB 101367), BM (SALA-L 4849), EA, ES \& RA (MACB 102365, 102366), ELL (BCN-Lich 17651, 17671),VA (VAL-Lich 27587), Qf; 14: BM (SALA-L 4798), EA, ES \& RA (MACB 101993), VA (VAL-Lich 27605), Js

Arthonia apatetica (A. Massal.) Th. Fr. - 8: ELL (BCN.-Lich 17474), Jc.

* $\neq$ Arthonia clemens (Tul.) Th. Fr. - 7: SPO (SPO-1552), L (Rhizoplaca melanophthalma).

₹ Arthonia epiphyscia Nyl. - 11: SPO (SP0-1563), L (Xanthoria parietina).

$\neq$ Arthonia subfuscicola (Linds.) Triebel - 14: VA (VAL-Lich 27606), L (Lecanora carpinea).

₹ Arthonia urceolata (Elenkin) Calat., Barreno \& V. J. Rico - 5: VA (VAL-Lich 27469), L (Circinaria hispida); 11: ELS (SANT 11663), L (C. hispida); 14: VA (VAL-Lich 27607), L (C. hispida).

\#Arthonia varians (Davies) Nyl. - 8: ELL (BCN-Lich 17497, 17506), SFB \& XLL (BCN-Lich 17725), L (Lecanora leptyrodes, L. cenisia); 10: SPO (SP0-1578), L (Lecanora rupicola); 12: MV \& VJR (MAF-Lich 16858), L (Lecanora rupicola).

Aspicilia cheresina (Müll. Arg.) Hue - 11: MV \& VJR (MAF-Lich 16846), Rcz.

Aspicilia contorta subsp. hoffmanniana S. Ekman \& Fröberg ex R. Sant. - 1: MV \& VJR (MAF-Lich 16742, 16744), Ys; 5: ELS (SANT 11856), VA (VAL-Lich 27470), Rcz, 14: ELL (BCN-Lich 17710), Rcz.

Aspicilia desertorum (Kremp.) Mereschk. - 14: BM (SALA-L 4844), Rcz.

Athallia alnetorum (Giralt, Nimis \& Poelt) Arup, Frödén \& Søchting (= Caloplaca alnetorum Giralt, Nimis \& Poelt) - 1: ELL (BCNLich 17356, 17359, 17362), Gh, Ot, Ssp; 4: ELL (BCN-Lich 17393), Qi; 5: ELL (BCN-Lich 17411, 17413, 17414, 17419, 17420, 17421, 17422, 17428), Jt, Pd, Tsp; 6: ELL (BCN-Lich 17434), Jt; 8: ELL (BCN-Lich 17486, 17497), Am, Psp; 11: ELL (BCN-Lich 17614, 17633), Qf, Qi; 13: ELL (BCN-Lich 17661, 17669, 17670, 17672), Qf; 14: ELL (BCN-Lich 17679, 17685, 17694), Bh, Jc, Js.

Athallia cerinella $(\mathrm{Nyl}$.) Arup, Frödén \& Søchting (= Caloplaca cerinella (Nyl.) Flagey) - 5: ELL (BCN-Lich 17428), Pd; 14: BM (SALA-L 4804), Jt.

Athallia holocarpa (Hoffm.) Arup, Frödén \& Søchting (= Caloplaca holocarpa (Hoffm.) A.E. Wade) - 1: ELL (BCN-Lich 17356, 17370, 17359, 17360), Gh, Ot, Ssp; 4: ELL (BCN-Lich 17384, 17386, 17387, 17390, 17393, 17396), Qi, Qp; 5: ELL (BCNLich 17416, 17417, 17419, 17424, 17428), ELS (SANT 11603), VA (VAL-Lich 27474), Jt, Pd, Qc, Rcz; 11: BM (SALA-L 4949), Rcz; 12: VA (VAL-Lich 27574), Qi; 14: VA (VAL-Lich 27611), $\mathrm{Bh}$.

* $\neq$ Athelia arachnoidea (Berk.) Jülich - 11: SPO (SPO-1577), L (Xanthoria parietina).

Bacidia igniarii (Nyl.) Oxne - 11: ELL (BCN-Lich 17625), Qi.

Bagliettoa calciseda (DC.) Gueidan \& Cl. Roux (=Verrucaria calciseda DC.) - 11: BM (SALA-L 4775), Rcz; 14: BM (SALA-L 4808), Rcz.

Bagliettoa marmorea (Scop.) Gueidan \& Cl. Roux (= Verrucaria marmorea (Scop.) Arnold) - 5: BM (SALA-L 5034), EA, ES \& RA (MACB 102311), Rcz; 11: BM (SALA-L 4940), Rcz.
** Bagliettoa parmigera (J. Steiner) Vězda \& Poelt (= Verrucaria parmigera J. Steiner) - 11: BM (SALA-L 4947), Rcz.

*Biatora subduplex (Nyl.) Printzen - 9: ELL (BCN-Lich 17549, 17550, 17554), Ps.

*Bilimbia lobulata (Sommerf.) Hafellner \& Coppins - 10: MR (MALich 16404), SM (MA-Lich 16354), Rcc.

Blastenia ferruginea (Huds.) A. Massal. (= Caloplaca ferruginea (Huds.) Th. Fr.) - 8: ELL (BCN-Lich 17506), Am; 10: ELL (BCNLich 17580), Qp; 12: SM (MA-Lich 16368), Ps; 13: ELL (BCNLich 17662, 17668), Qf; 14: BM (SALA-L 4805), Jt.

Blastenia hungarica (H. Magn.) Arup, Søchting \& Frödén (= Caloplaca hungarica $\mathrm{H}$. Magn.) - 2: ELL (BCN-Lich 17376, 17381), Pp; 4: ELL (BCN-Lich 17402, 17404, 17405, 17410), Mm; 6: ELL (BCN-Lich 17443, BCN-Lich 17444), Ps; 8: ELL (BCNLich 17468, 17475), Jc.

*Brodoa intestiniformis (Vill.) Goward - 10: BM (SALA-L 4991), ELS (SANT 11857), MV \& VJR (MAF-Lich 16831), Rcc.

Bryoria capillaris (Ach.) Brodo \& D. Hawksw. - 9: EA, ES \& RA (MACB 102118, 102119, 102120), ELS (SANT 11610), MR (MALich 16390), MV \& VJR (MAF-Lich 16812, 16813, 16820), VA (VAL-Lich 27516), Ps.

Bryoria fuscescens (Gyeln.) Brodo et D. Hawksw. - 8: EA, ES \& RA (MACB 102227, 102228), ELS (SANT 11609), ELL (BCN-Lich 17458), SFB \& XLL (BCN-Lich 17720), SPO (SPO-1605), Ps, Qp, Rcc, Smu; 9: BM (SALA-L 5025), EA, ES \& RA (MACB 102168), ELS (SANT 11611), MR (MA-Lich 16389), VA (VALLich 27517), Ps; 10: ELL (BCN-Lich 17568), Ps.

Bryoria implexa (Gyeln.) Brodo \& D. Hawksw. - 9: EA, ES \& RA (MACB 102170), ELL (BCN-Lich 17544, 17548), MR (MA-Lich 16388), Ps.

Buellia cedricola Werner - 14: BM (SALA-L 4796) Jc.

**Buellia chloroleuca Körb. - 9: SPO (SPO-1612), Mm.

Buellia disciformis (Fr.) Mudd - 14 SM (MA-Lich 16376), Js.

Buellia erubescens Arnold - 8: ELL (BCN-Lich 17463, 17464), Ps; 10: ELL (BCN-Lich 17569, 17587, 17595, 17598), Ps, Mm; 12: ELL (BCN-Lich 17639, 17640), Ps.

Buellia griseovirens (Turner \& Borrer ex Sm.) Almb. - 4: ELL (BCNLich 17409), Mm; 9: ELL (BCN-Lich 17555), SPO (SPO-1610), Mm; 10: ELL (BCN-Lich 17563, 17581, 17597), Ps, Qp; 12: ELL (BCN-Lich 17636), Ps; 14: BM (SALA-L 4797 Jc.

Buellia triseptata A. Nordin - 14: MR (MA-Lich 16430), Js.

*Buellia zoharyi Galun - 1: BM (SALA-L 4966), Ys.

Calicium corynellum (Ach.) Ach. - 10: SFB \& XLL (BCN-Lich 17748), Rcc.

*Calogaya arnoldii (Wedd.) Arup, Frödén \& Søchting (= Caloplaca arnoldii (Wedd.) Zahlbr. ex Ginzb.) - 8: JM (P08830L), Rcc.

Calogaya biatorina (A. Massal.) Arup, Frödén \& Søchting (= Caloplaca biatorina (Trevis.) J. Steiner) - 5: VA (VAL-Lich 27471), Rcz; 11: MV \& VJR (MAF-Lich 16844), VA (VAL-Lich 27541), Rcz.

Calogaya pusilla (A. Massal.) Arup, Frödén \& Søchting (= Caloplaca saxicola (Hoffm.) Nordin) - 14: BM (SALA-L 4803), Rcz.

**Calogaya schistidii (Anzi) Arup, Frödén \& Søchting (= Caloplaca schistidii (Anzi) Zahlbr.) - 5: EA, ES \& RA (MACB 102296, 102297), ELS (SANT 11624), JM (PO8844L), Mu; 6: SPO (SPO1547), Mu; 8: JM (PO8843L), 11: SM (MA-Lich 16361), Rcz; Mu; 14: ELS (SANT 11627), JM (P08842L), MR (MA-Lich 16427), MV \& VJR (MAF-Lich 16867), Mu, Rcz.

Caloplaca aractina (Fr.) Häyrén - 12: JM (P08829L), Rcc.

*Caloplaca arnoldii subsp. obliterata (Pers.) Gaya - 4: JM (P08831L), Rrd. 
*Caloplaca biatorina var. baumgartneri (Zahlbr.) Poelt - 7: MV \& VJR (MAF-Lich 16786), Rcc.

Caloplaca cerina (Ehrh. ex Hedw.) Th. Fr. var. cerina - 4: ELL (BCNLich 17389, 17393), Qi; 5: ELL (BCN-Lich 17413, 17416, 17419, 17421, 17423, 17424, 17428, 17430, 17431), VA (VAL-Lich 27472), Jt, Pd, Qc, Sa, Tsp; 6: ELL (BCN-Lich 17434), Jt; 8: ELL (BCN-Lich 17476) Cm; 11: ELL (BCN-Lich 17622, 17633), BM (SALA-L 4764), Qf, Qi; 13: BM (SALA-L 4865), Qf; 14: BM (SALA-L 4795), Jt.

*Caloplaca cerina var. chloroleuca (Sm.) Th. Fr. - 6: VA (VAL-Lich 27497), Jt.

*Caloplaca chlorina (Flot.) Sandst. - 2: JM (P08832L), Rrd; 7: MV \& VJR (MAF-Lich 16788), Rcc; 8: JM (P08833L), Rcc.

**Caloplaca congrediens (Nyl.) Zahlbr. - 12: JM (PO8834L), Rcc.

\#Caloplaca grimmiae (Nyl.) H. Olivier - 12: MV \& VJR (MAF-Lich 16854), L (Candelariella sp.).

Caloplaca haematites (Chaub. ex St.-Amans) Zwackh-1: ELL (BCNLich 17356, 17370, 17357, 17359, 17360, 17361, 17362), VA (VAL-Lich 27453), SPO (SPO-1597), Ot, Ssp, Tsp; 5: ELL (BCNLich 17412, 17413, 17414, 17420, 17422), VA(VAL-Lich 27473), Jt, Tsp; 6: ELL (BCN-Lich 17434), Jt; 13: ELL (BCN-Lich 17661), Qf; 14: ELL (BCN-Lich 17675), Js.

**Caloplaca irrubescens (Arnold) Zahlbr. - 2: SFB \& XLL (BCNLich 17716), Rcc.

**Caloplaca lacteoides Nav.-Ros. \& Hladún - 5: ELS (SANT 11612), Rcz.

Caloplaca lucifuga G. Thor - 11: ELL (BCN-Lich 17622), Qi; 14: ELL (BCN-Lich 17685), Js.

**Caloplaca muscorum (A. Massal.) M. Choisy \& Werner - 4: JM (P08835L), Mu; 7: MV \& VJR (MAF-Lich 16772) Rcc, 8: SFB \& XLL (BCN-Lich 17726), Mu.

Caloplaca teicholyta (Ach.) J. Steiner-14: BM (SALA-L 4802), Rcz.

Caloplaca ulcerosa Coppins \& P. James - 4: ELL (BCN-Lich 17387), Qp; 5: ELL (BCN-Lich 17413, 17415, 17424), Jt, Qc, Tsp.

Candelaria concolor (Dicks.) Arnold - 5: ELL (BCN-Lich 17432), Sa; 6: ELL (BCN-Lich 17442), Ps.

Candelariella aurella (Hoffm.) Zahlbr. - 1: BM (SALA-L 4974), EA, ES \& RA (MACB 102004), ELS (SANT 11613), Ys; 11: BM (SALA-L 4948), Rcz; 14: ELL (BCN-Lich 17674, 17675, 17676, 17709), MV \& VJR (MAF-Lich 16862), Mm, Rcz.

Candelariella faginea Nimis, Poelt \& Puntillo - 13: BM (SALA-L 4884), Qf.

**Candelariella viae-lacteae $\mathrm{G}$. Thor \& V. Wirth - 1: ELL (BCN-Lich 17370, 17357, 17359, 17360, 17361, 17362), Gh, Ot; 5: ELL (BCN-Lich 17413, 17414), Tsp.

Candelariella vitellina (Ehrh.) Müll. Arg. - 1: BM (SALA-L 4970), VA (VAL-Lich 27453), Ot, Ys; 4: ELL (BCN-Lich 17404, 17408), Mm; 5: ELL (BCN-Lich 17417, 17432), Qc, Sa; 8: ELL (BCN-Lich 17456, 17464, 17466, 17467, 17468, 17471, 17474, $17476,17485,17507,17508,17517,17526)$, SFB \& XLL (BCNLich 17731), Cm, Jc, Ps, Psp, Qp, Rcc; 9: ELL (BCN-Lich 17556), Mm; 10: BM (SALA-L 4983), ELL (BCN-Lich 17581, 17596, 17598), ELS (SANT 11614), MV \& VJR (MAF-Lich 16840), Ps, Qp, Rcc; 11: BM (SALA-L 4964), Qi; 12: EA, ES \& RA (MACB 102359), ELL (BCN-Lich 17649), Qp, Rcc; 13: BM (SALA-L 4883), ELL (BCN-Lich 17662), Qf; 14: BM (SALA-L 4806), ELL (BCN-Lich 17681, 17685, 17687, 17694), Bh, Jc, Js, Mm.

Candelariella xanthostigma (Pers. ex Ach.) Lettau - 1: ELL (BCNLich 17360, 17362), Gh, Ot; 4: ELL (BCN-Lich 17389, 17390,
17392, 17393, 17396), Qi, Qp; 8: ELS (SANT 11658), Qp; 13: ELL (BCN-Lich 17672), Qf; 14: BM (SALA-L 4827), Jc.

* $\neq$ Carbonea vitellinaria (Nyl.) Hertel - 10: ELS (SANT 11615), L (Candelariella vitellina).

**Catapyrenium daedaleum (Kremp.) Stein - 14: ELL (BCN-Lich 17708), Mu.

Catapyrenium squamulosum (Ach.) Breuss - 1: BM (SALA-L 4969), Ys; 5: EA, ES \& RA (MACB 102316, 102317), ELS (SANT 11616), Rcz, Sue; 11: BM (SALA-L 4935), Sue; 14: BM (SALAL 4814), Sue.

Catillaria chalybeia (Borrer) A. Massal. - 4: SPO (SPO-1554), Rrd.

Catinaria atropurpurea (Schaer.) Vězda \& Poelt-1: ELL (BCN-Lich 17359), Gh.

* $\neq$ Cercidospora melanophtalmae Nav.-Ros. \& Calat. - 7: SPO (SPO1530), L (Rhizoplaca melanophthalma).

Cetraria aculeata (Schreb.) Fr. - 4: ARB (MACB 101372), EA, ES \& RA (MACB 102032), Sue; 6: EA, ES \& RA (MACB 102321, 102322), ELL (BCN-Lich 17449), MV \& VJR (MAF-Lich 16770), VA (VAL-Lich 27499, 27500), Sue; 7: ARB (MACB 101373), Sue; 9: EA, ES \& RA (MACB102186); Sue; 10: ARB (MACB 101374), BM (SALA-L 4984), Sue; 11: BM (SALA-L 4776), EA, ES \& RA (MACB 102084), MR (MA-Lich 16409), Sue; 12: EA, ES \& RA (MACB 102354, 102355), ELS (SANT 11662), Sue; 14: BM (SALA-L 4815, 4816), EA, ES \& RA (MACB 101991), ELL (BCN-Lich 17697), SM (MA-Lich 16375), VA (VAL-Lich 27613), Js, Sue.

Cetraria islandica (L.) Ach. - 10: MR (MA-Lich 16397), SM (MALich 16357), Sue.

**Cetraria muricata (Ach.) Eckfeldt -3: ARB (MACB 101376), Sue; 6: ARB (MACB 101375), Sue; 11: VA (VAL-Lich 27545), Sue.

Circinaria alpicola (Elenkin) Sohrabi (= Aspicilia fruticuloso-foliacea (Elenkin) Sohrabi) - 11: ELS (SANT 11607), Sue; 14. JM (P08826L), Sue.

Circinaria caesiocinerea (Nyl. ex Malbr.) A. Nordin, Saviç \& Tibell (= Aspicilia caesiocinerea (Nyl. ex Malbr.) Arnold) - 10: SFB \& XLL (BCN-Lich 17743), Rcc.

Circinaria calcárea (L.) A. Nordin, Saviç \& Tibell (= Aspicilia calcarea (L.) Körb.) - 1: BM (SALA-L 4980) Ys; 5: ARB (MACB 101368), VA (VAL-Lich 27470), Rcz; 11: BM (SALA-L 4945), VA(VAL-Lich 27543), Rcz; 14: BM (SALA-L 4801), MV \& VJR (MAF-Lich 16870), VA (VAL-Lich 27608), Rcz.

Circinaria contorta (Hoffm.) A. Nordin, Saviç \& Tibell (= Aspicilia contorta (Hoffm.) Körb.) - 1: BM (SALA-L 4972), ELL (BCNLich 17361, 17366), Ot, Ys; 6: ELL (BCN-Lich 17452, 17454), Rcc; 11: BM (SALA-L 4941), Rcz; 14: JM (P08823L), Rcz.

**Circinaria crespiana (V. J. Rico) Sohrabi \& V. J. Rico (= Aspicilia crespiana V.J. Rico) - 4: JM (P08824L), Rrd; 7: MV \& VJR (MAF-Lich 16789), SPO (SPO-1499), Smu, Rcc.

Circinaria gyrosa Sohrabi, Sipman, V. John \& V. J. Rico - 5: MV \& VJR (MAF-Lich 16766), Sue; 6: BM (SALA-L 5037), ELS (SANT 11606), Sue; 11: ARB (MACB 101369), BM (SALA-L 4760, 4930), EA, ES \& RA (MACB 102047, 102048), VA (VALLich 27544), Sue; 14: BM (SALA-L 4788), EA, ES \& RA (MACB 101992), ELL (BCN-Lich 17698), JM (P08825L), MR (MA-Lich 16436), SM (MA-Lich 16382), VA (VAL-Lich 27609), Sue.

Circinaria hispida (Mereschk.) A. Nordin, S. Saviç \& Tibell (= Aspicilia hispida Mereschk.) - 5: ARB (MACB 101370), BM (SALAL 5033), EA, ES \& RA (MACB 102295), MV \& VJR (MAF-Lich 16765), Sue; 6: BM (SALA-L 5036), EA, ES \& RA (MACB 102320), Sue; 11: ARB (MACB 101371), BM (SALA-L 4761), 
EA, ES \& RA (MACB 102046), ELS (SANT 11608), JM (P08828L), SM (MA-Lich 16362), Sue; 14: BM (SALA-L 4789, 4843), JM (P08827L), VA (VAL-Lich 27604), Sue.

**Cladonia asahinae J.W. Thomson - 9: EA, ES \& RA (MACB 102187), Sue.

Cladonia carneola (Fr.) Fr. - 9: EA, ES \& RA (MACB 102189, 102191), Mm.

Cladonia cervicornis (Ach.) Flot. - 2: ARB (MACB 101566), Sue.

Cladonia chlorophaea (Flörke ex Sommerf.) Spreng. - 10: ARB (MACB 101438), Sue.

Cladonia coniocraea (Flörke) Spreng. - 3: SM s/n, Sue; 4: ARB (MACB 101437), EA, ES \& RA (MACB 102038), Ba; 9: ELL (BCN-Lich 17555, 17557), VA (VAL-Lich 27518), Ba; 10: ARB (MACB 101436), ELL (BCN-Lich 17567, 17585, 17586), MR (MA-Lich 16408), Ba.

Cladonia convoluta (Lamkey) Cout. - 1: ARB (MACB 101435), EA, ES \& RA (MACB 102005), ELL (BCN-Lich 17363), VA (VALLich 27454), Ys; 5: VA (VAL-Lich 27475), Sue; 6: ARB (MACB 101434), EA, ES \& RA (MACB 102328), ELL (BCN-Lich 17450), ELS (SANT 11618), VA (VAL-Lich 27501), Sue; 11: EA, ES \& RA (MACB 102087), VA (VAL-Lich 27546), Sue; 14: BM (SALA-L 4838), EA, ES \& RA (MACB 101998), ELL (BCN-Lich 17702), VA (VAL-Lich 27614), Sue.

Cladonia cyathomorpha Stirt. ex Walt. Watson - 4: EA, ES \& RA (MACB 102037), Sue; 8: EA, ES \& RA (MACB 102257), Sue; 10: ARB (MACB 101433), Sue.

Cladonia diversa Asperges - 10: ARB (MACB 101432), Sue.

Cladonia fimbriata (L.) Fr. - 4: ARB (MACB 101427), ELL (BCNLich 17397, 17406, 17409), Ba, Mm, Sue; 9: ELL (BCN-Lich 17543, 17559), VA (VAL-Lich 27519), Mm, Ba; 10: ELL (BCNLich 17587), Mm.

Cladonia firma (Nyl.) Nyl. - 4: ARB (MACB 101431), EA, ES \& RA (MACB 102039, 102040), Sue.

Cladonia foliacea (Huds.) Willd. - 1: MV \& VJR (MAF-Lich 16745, 16750), Ys; 4: ARB (MACB 101430), EA, ES \& RA (MACB 102041), Sue; 9: EA, ES \& RA (MACB 102176), Sue; 10: BM (SALA-L 4986), EA, ES \& RA (MACB 102111), ELL (BCNLich 17599), Sue; 11: BM (SALA-L 4768), SM (MA-Lich 16364), Sue; 12: VA (VAL-Lich 27562), Rcc; 14: BM (SALAL 4799), Sue.

Cladonia furcata (Huds.) Schrad. - 4: ARB (MACB 101429), EA, ES \& RA (MACB 102042), Sue; 6: VA (VAL-Lich 27502), Sue; 10: ARB (MACB 101428), Sue; 11: BM (SALA-L 4767), VA (VALLich 27547), Sue.

Cladonia glauca Flörke - 4: ARB (MACB 101426), EA, ES \& RA (MACB 102043, 102044, 102284, 102285), Ba, Mm, Sue; 9: EA, ES \& RA (MACB 102192), Mm.

*Cladonia gracilis (L.) Willd. - 10: ARB (MACB 101425), Rcc.

*Cladonia homosekikaica Nuno - 2: ARB (MACB 101424), Ba; 4: ARB (MACB 101423), EA, ES \& RA (MACB 102286), Ba, Sue; 9: EA, ES \& RA (MACB 102188), Mm; 10: ELL (BCN-Lich 17585), Mm

Cladonia humilis (With.) J.R. Laundon - 4: ARB (MACB 101422), EA, ES \& RA (MACB 102287), Sue.

Cladonia macilenta Hoffm. - 4: ARB (MACB 101421), Ba.

Cladonia mitis Sandst. - 10: MR (MA-Lich 16398), SM (MA-Lich 16347), Sue.

Cladonia pocillum (Ach.) O. J. Rich. -1: ELL (BCN-Lich 17358) MuYs; 2: ARB (MACB 101420), EA, ES \& RA (MACB 102015), Sue; 4: ARB (MACB 101419), EA, ES \& RA (MACB 102288),
ELL (BCN-Lich 17395), Ba, Sue; 5: VA (VAL-Lich 27476), Sue; 8: EA, ES \& RA (MACB 102269), Sue; 14: ELL (BCN-Lich 17705), Sue.

**Cladonia pseudopityrea Vain. - 4: ARB (MACB 101418), Sue.

**Cladonia pulvinella S. Hammer - 4: ARB (MACB 101417), Sue.

Cladonia pyxidata (L.) Hoffm. - 2: EA, ES \& RA (MACB 102014), Sue-Rrd; 4: ARB (MACB 101416, 101499), Sue; 10: ELL (BCNLich 17601), Sue.

*Cladonia ramulosa (With.) J. R. Laundon-4: ARB (MACB 101412), EA, ES \& RA (MACB 102289), Sue.

Cladonia rangiformis Hoffm. - 1: EA, ES \& RA (MACB 102006), MV \& VJR (MAF-Lich 16751), Ys; 4: ARB (MACB 101415), EA, ES \& RA (MACB 102290, 102291, 102292), ELL (BCN-Lich 17383), Sue; 6: ARB (MACB 101414), EA, ES \& RA (MACB 102327), Sue; 8: ELL (BCN-Lich 17531), Sue; 9: EA, ES \& RA (MACB 102193), Mm; 10: BM (SALA-L 4987), EA, ES \& RA (MACB 102110), ELL (BCN-Lich 17600), Sue; 11: MR (MALich 16415), SM (MA-Lich 16363), Sue; 12: EA, ES \& RA (MACB 102360), Sue; 14: ARB (MACB 101413), BM (SALA-L 4837), ELL (BCN-Lich 17700), Rcc, Sue.

**Cladonia rei Schaer. - 4: ELL (BCN-Lich 17395), Ba; 9: ELL (BCNLich 17558, 17559), Mm.

Cladonia subrangiformis (L.) Scriba ex Sandst. - 4: EA, ES \& RA (MACB 102293), Sue; 6: ARB (MACB 101410, 101409), EA, ES \& RA (MACB 102324, 102325, 102326), ELL (BCN-Lich 17451), MV \& VJR (MAF-Lich 16771), Sue; 10: MR (MA-Lich 16399), SM (MA-Lich 16348), Sue; 11: ARB (MACB 101408, 101407), EA, ES \& RA (MACB 102088), Sue; 14: EA, ES \& RA (MACB 102002), Sue.

Cladonia subulata (L.) Weber ex F. H. Wigg. - 2: ELL (BCN-Lich 17373), Smu; 4: ELL (BCN-Lich 17409), Mm; 9: EA, ES \& RA (MACB 102190), Mm; 10: ARB (MACB 101406), Sue.

Cladonia symphycarpa (Flörke) Fr. - 4: ARB (MACB 101405), Sue; 14: BM (SALA-L 4839), Sue.

* $\neq$ Clypeococcum epicrassum (H. Olivier) Hafellner \& Nav.-Ros. - 5: SPO (SPO-1589), L (Squamarina cartilaginea).

*Collema coccophorum Tuck. - 5: ELS (SANT 11619), Sue; 14: JM (P08838L), Rcz.

Collema crispum (Huds.) Weber ex F. H. Wigg. - 11: BM (SALA-L 4770), Sue.

Collema cristatum (L.) Weber ex F. H. Wigg. var. cristatum - 1: BM (SALA-L 4973) Ys; 5: EA, ES \& RA (MACB 102314, 102315), ELS (SANT 11620), VA (VAL-Lich 27477), Rcz, Smu, Sue; 6: ELS (SANT 11621), Sue; 11: BM (SALA-L 4938), Rcz.

Collema cristatum var. marginale (Huds.) Degel. - 14: VA (VAL-Lich 27615), Rcz.

Collema flaccidum (Ach.) Ach. - 1: ELL (BCN-Lich 17367), Ys.

Collema furfuraceum Du Rietz-11: ELL (BCN-Lich 17607), Qi; 13: BM (SALA-L 4874); ELL (BCN-Lich 17654, 17655, 17662), VA (VAL-Lich 27588), Qf.

**Collema polycarpon subsp. corcyrense (Arnold) Pišút - 14: JM (P08839L), Rcz.

Collema subflaccidum Degel. - 1: ELL (BCN-Lich 17361), Ot; 8: ELL (BCN-Lich 17507), Qp; 13: BM (SALA-L 4875), MR (MA-Lich 16420), SM (MA-Lich 16371), Qf.

Collema tenax (Sw.) Ach. var. tenax - 5: ELL (BCN-Lich 17425), Sue; 11: BM (SALA-L 4769), Sue; 14: ELL (BCN-Lich 17699, 17707, 17709), Sue.

** Collema tenax var. vulgare (Schaer.) Degel. - 14: VA (VAL-Lich 27616), Rcz. 
Collema undulatum Laurer ex Flot. - 14: BM (SALA-L 4807), Jc. *Cornicularia normoerica (Gunnerus) Du Rietz - 7: SPO (SPO-1500), Rcc; 8: EA, ES \& RA (MACB 102256, 102262), ELL (BCN-Lich 17535), SFB \& XLL (BCN-Lich 17731), Rcc; 10: BM (SALA-L 4985), EA, ES \& RA (MACB 102101), MR (MA-Lich 16403), MV \& VJR (MAF-Lich 16828), SFB \& XLL (BCN-Lich 17747), SM (MA-Lich 16353), VA (VAL-Lich 27529), Rcc.

**Cyphelium lecideinum (Nyl.) Trevis. - 10: SPO (SPO-1591), Rcc.

** $\neq$ Cyphelium marcianum B. de Lesd. - 8: SFB \& XLL (BCN-Lich 17723), L (Pertusaria pseudocorallina).

** $\neq$ Dactylospora parasitica (Flörke) Zopf - 8: SPO (SPO-1555), SFB \& XLL (BCN-Lich 17733), Rcc, L (Pertusaria pertusa var. rupestris).

Dermatocarpon miniatum (L.) W. Mann - 2: ARB (MACB 101377), Rrd; 4: EA, ES \& RA (MACB 102030), Rrd; 10: BM (SALA-L 4988), Rcc; 14: MV \& VJR (MAF-Lich 16866), Rcz.

Dimelaena oreina (Ach.) Norman - 10: ARB (MACB 101378), SFB \& XLL (BCN-Lich 17749), Rcc; 12: VA (VAL-Lich 27563), Qi.

**Diploicia canescens (Dicks.) A. Massal. - 1: BM (SALA-L 4979), Ys.

Diploschistes diacapsis (Ach.) Lumbsch - 14: BM (SALA-L 4818), ELL (BCN-Lich 17695), Sue.

Diploschistes gypsaceus (Ach.) Zahlbr. - 5: VA (VAL-Lich 27478), Rcz; 6: VA (VAL-Lich 27503), Rcz.

Diploschistes muscorum (Scop.) R. Sant. - 2: ARB (MACB 101379), Sue; 4: VA (VAL-Lich 27465), Rrd; 6: ELS (SANT 11623), Mu; 8: SFB \& XLL (BCN-Lich 17728, 17736), Cl; 10: BM (SALA-L 4989), Sue.

Diploschistes ocellatus (Fr.) Norman - 6: ELS (SANT 11622), MV \& VJR (MAF-Lich 16768), SPO (SP0-1599), Rcz, Sue.

Diploschistes scruposus (Schreb.) Norman - 4: SPO (SPO-1556), Rrd; 7: MV \& VJR (MAF-Lich 16804), Rcc; 10: SFB \& XLL (BCNLich 17739, 17741, 17743), Rcc.

Diplotomma alboatrum (Hoffm.) Flot. - 1: ELL (BCN-Lich 17370, 17357, 17362, 17365), Ot, Ys; 2: SFB \& XLL (BCN-Lich 17716), Rrd; 5: ELL (BCN-Lich 17420, 17421, 17422, 17424), MV \& VJR (MAF-Lich 16763), Jt, Rcz; 6: ELL (BCN-Lich 17434), Jt; 13: BM (SALA-L 4885), ELL (BCN-Lich 17669, 17670), Qf; 14: MV \& VJR (MAF-Lich 16868), Rcz.

**Diplotomma epipolium (Ach.) Arnold - 5: ARB (MACB 101380), SPO (SPO-1608), Rcz.

***Diplotomma hedinii (H. Magn.) P. Clerc \& Cl. Roux - 5: VA (VALLich 27479), Rcz; 14: ELL (BCN-Lich 17709), VA (VAL-Lich 27617), Rcz.

**Diplotomma rivas-martinezii (Barreno \& A. Crespo) Barreno \& A. Crespo - 1: MV \& VJR (MAF-Lich 16743), Ys.

Endocarpon pusillum Hedw. - 5: ARB (MACB 101504), Sue; 11: BM (SALA-L 4965), Sue; 13: BM (SALA-L 4893), Sue.

* $\neq$ Endococcus perpusillus Nyl. - 10: SPO (SPO-1582), L (Aspicilia $\mathrm{sp}$.).

**Epigloea soleiformis Döbbeler - 7: SPO (SPO-1550), Hongo alguícola.

Evernia prunastri (L.) Ach. - 2: ELL (BCN-Lich 17375), Mm; 4: ELL (BCN-Lich 17397), Pp; 6: ELL (BCN-Lich 17439), Ps; 8: BM (SALA-L 4617), EA, ES \& RA (MACB 102241), ELS (SANT 11664), Am, Qp; 10: MR (MA-Lich 16394), SM (MA-Lich 16342), Qp; 11: BM (SALA-L 4779), EA, ES \& RA (MACB 102078), ELL (BCN-Lich 17626, 17634), MV \& VJR (MAF-Lich 16852), VA (VAL-Lich 27548), Qf, Qi; 12: VA (VAL-Lich 27564), Qi.
Fuscopannaria mediterranea (Tav.) P. M. Jørg. - 7: MV \& VJR (MAFLich 16776), Mu; 8: BM (SALA-L 4645), Qp.

Gyalolechia bracteata (Hoffm.) A. Massal. (= Fulgensia bracteata (Hoffm.) Räsänen) - 1: BM (SALA-L 4968), Ys.

Gyalolechia flavorubescens (Huds.) Søchting, Frödén \& Arup (= Caloplaca flavorubescens (Huds.) J.R. Laundon) - 13: BM (SALAL 4860), Qf; 14: BM (SALA-L 4825), Jt.

Gyalolechia fulgens (Sw.) Søchting, Froden \& Arup (= Fulgensia fulgens (Sw.) Elenkin) - 1: BM (SALA-L 4967), EA, ES \& RA (MACB 102007), ELL (BCN-Lich 17364, 13768), JM (PO8840L), Ys; 5: EA, ES \& RA (MACB 102310), Sue; 6: ELS (SANT 11625), Rcz.

Gyalolechia fulgida (Nyl.) Søchting, Froden \& Arup (= Fulgensia fulgida (Nyl.) Szatala) - 5: VA (VAL-Lich 27481), Sue; 6: JM (P08841L), Rcz.

Hyperphyscia adglutinata (Flörke) H. Mayrhofer \& Poelt - 5: ELL (BCN-Lich 17415), Qc; 8: BM (SALA-L 4609), Am.

Hypocenomyce scalaris (Ach. ex Lilj.) M. Choisy - 9: BM (SALA-L 5026), EA, ES \& RA (MACB 102175), JM (P08845L), MV \& VJR (MAF-Lich 16818), VA (VAL-Lich 27520), Ps.

Hypogymnia farinacea Zopf - 4: ELL (BCN-Lich 17401, 17403), Mm, Pp; 8: BM (SALA-L 4614),Qp; 9: BM (SALA-L 5027), EA, ES \& RA (MACB 102177, 102178, 102179), ELL (BCN-Lich 17546, 17549, 17554), JM (P08846L), MV \& VJR (MAF-Lich 16811), SM (MA-Lich 16336), VA (VAL-Lich 27521), Ps; 10: BM (SALA-L 4990), ELL (BCN-Lich 17560, 17562, 17564, 17566, 17568, 17583), MR (MA-Lich 16405), Ps, Qp; 12: ELL (BCNLich 17636, 17639, 17643, 17650), Ps, Qp.

Hypogymnia physodes (L.) Nyl. - 8: BM (SALA-L 4615), ELS (SANT 11665), Ps, Qp; 9: BM (SALA-L 5028), EA, ES \& RA (MACB 102182), ELL (BCN-Lich 17545), JM (P08847L), MR (MA-Lich 16387), MV \& VJR (MAF-Lich 16817), SM (MA-Lich 16337), VA (VAL-Lich 27522), Ps; 14: VA (VAL-Lich 27618), Bh.

Hypogymnia tubulosa (Schaer.) Hav. - 4: ARB (MACB 101381), EA, ES \& RA (MACB 102029), Qi; 8: BM (SALA-L 4616), EA, ES \& RA (MACB 102255), ELL (BCN-Lich 17457, 17458, 17460, 17461, 17462, 17465, 17473, 17522), Jc, Ps, Qp; 9: BM (SALAL 5029), ELL (BCN-Lich 17554), JM (P08847L), VA (VAL-Lich 27523), Ps; 10: BM (SALA-L 4992), ELL (BCN-Lich 17562 , 17565, 17573), ELS (SANT 11626), Ps, Qp, Rcc; 11: EA, ES \& RA (MACB 102086, 102341), ELL (BCN-Lich 17627), ELS (SANT 11628), MV \& VJR (MAF-Lich 16851), VA (VAL-Lich 27549), Qi; 12: VA (VAL-Lich 27565), Qi; 14: BM (SALA-L 4839), VA (VAL-Lich 27619), Bh.

Immersaria athroocarpa (Ach.) Rambold \& Pietschm. - 4: ARB (MACB 101382), Rrd.

Imshaugia aleurites (Ach.) S. L. F. Mey. - 2: EA, ES \& RA (MACB 102016), Pp; 4: ARB (MACB 101383), EA, ES \& RA (MACB 102294), ELL (BCN-Lich 17400, 17401), Pp.

* $\neq$ Intralichen lichenicola (M. S. Christ. \& D. Hawksw.) D. Hawksw. \& M. S. Cole - 5: VA (VAL-Lich 27471), L (Caloplaca biatori$n a)$.

Lasallia hispanica (Frey) Sancho \& A. Crespo - 3: ARB (MACB 101384), VA (VAL-Lich 27460), Rrd; 7: MV \& VJR (MAF-Lich 16794), Rcc; 8: EA, ES \& RA (MACB 102236, 102237, 102238, 102239), ELL (BCN-Lich 17540), SFB \& XLL (BCN-Lich 17730 , 17735), Rcc; 10: SFB \& XLL (BCN-Lich 17746), Rcc; 12: EA, ES \& RA (MACB 102343), Rcc.

Lasallia pustulata (L.) Mérat - 2: ARB (MACB 101385), EA, ES \& RA(MACB 102008), SFB \& XLL (BCN-Lich 17715), Rrd; 4: EA, 
ES \& RA (MACB 102023), MV \& VJR (MAF-Lich 16759), VA (VAL-Lich 27466), Rrd; 10: BM (SALA-L 4993), EA, ES \& RA (MACB 102195), ELS (SANT 11666), MR (MA-Lich 16400), SM (MA-Lich 16350), Rcc; 12: EA, ES \& RA (MACB 102342), Rcc. Lecania cyrtellina (Nyl.) Sandst. - 13: BM (SALA-L 4886), Qf.

Lecania viridulogranulosa (Harm.) Zahlbr. - 1: ELL (BCN-Lich 17356, 17357, 17359, 17360), Gh, Ot, Ssp; 5: ELL (BCN-Lich 17414, 17419, 17420, 17421, 17424), Jt, Tsp; 6: ELL (BCN-Lich 17434, 17444), Jt, Ps; 10: ELL (BCN-Lich 17582), Qp; 13: ELL (BCN-Lich 17670).

**Lecanora achariana A. L. Sm. - 10: MV \& VJR (MAF-Lich 16827), Rcc.

*Lecanora albella (Pers.) Ach. - 8: ELS (SANT 11633), Qp.

Lecanora albellula (Nyl.) Th. Fr. - 10: ELL (BCN-Lich 17561), Ps.

Lecanora bolcana (Pollini) Poelt - 4: ELS (SANT 11859), Rrd; 7: SPO (SPO-1510), Rcc; 8: JM (P08848L), Rcc; 12: MV \& VJR (MAFLich 16855), Rcc.

Lecanora carpinea (L.) Vain. - 4: ELL (BCN-Lich 17386, 17387, 17391, 17396), Qi, Qp; 6: ELL (BCN-Lich 17444), Ps; 8: BM (SALA-L 4598), ELL (BCN-Lich 17458, 17462, 17463, 17464, 17468, 17475), Am, Jc, Ps; 10: ELL (BCN-Lich 17578, 17581, 17583, 17584), Jc, Qp; 11: BM (SALA-L 4783), VA (VAL-Lich 27550), Qi; 12: ELL (BCN-Lich 17640, 17647, 17650), VA (VALLich 27566), Ps, Qi, Qp; 13: BM (SALA-L 4880), ELL (BCNLich 17655, 17657, 17658, 17662, 17669), Qf; 14: ELL (BCNLich 17693), VA (VAL-Lich 27611), Bh, Psp.

*Lecanora cenisia Ach. - 8: SFB \& XLL (BCN-Lich 17725, 17731), Rcc; 10: MV \& VJR (MAF-Lich 16833), Rcc.

**Lecanora cenisia var. soredians Suza - 10: MV \& VJR (MAF-Lich 16837, 16834), Rcc.

Lecanora chlarotera Nyl. - 1: ELL (BCN-Lich 17359), Gh; 2: ELL (BCN-Lich 17376), Mm; 5: VA (VAL-Lich 27482), Jt; 6: ELL (BCN-Lich 17437, 17443), Jt, Ps; 8: BM (SALA-L 4605), Qp; 11: BM (SALA-L 4782), ELL (BCN-Lich 17605, 17613, 17616, 17618, 17619, 17633, 17634), VA (VAL-Lich 27550), Qf, Qi; 12: ELL (BCN-Lich 17648), SM (MA-Lich 16366), VA (VAL-Lich 27567), Ps, Qi, Qp; 13: BM (SALA-L 4881), ELL (BCN-Lich 17660, 17661), Qf; 14: BM (SALA-L 4800), ELL (BCN-Lich 17682), Jc.

Lecanora conizaeoides Nyl. ex Cromb. - 2: ELL (BCN-Lich 17380), $\mathrm{Mm}$

Lecanora crenulata Hook. - 1: SPO (SP0-1604), Tsp; 11: VA (VALLich 27551), Rcz.

Lecanora dispersa (Pers.) Röhl. - 5: SPO (SP0-1601), Rcz; 8: SFB \& XLL (BCN-Lich 17722), Rcc; 11: BM (SALA-L 4957), Rcz.

Lecanora expallens Ach. - 2: ELL (BCN-Lich 17374), Pp; 8: ELL (BCN-Lich 17464, 17467, 17474), Jc, Ps; 9: ELL (BCN-Lich 17557), Mm; 10: ELL (BCN-Lich 17589, 17590, 17592, 17593), Mm; 12: ELL (BCN-Lich 17640), Ps.

Lecanora gangaleoides Nyl. - 2: SFB \& XLL (BCN-Lich 17714), Rrd.

**Lecanora garovaglioi (Körb.) Zahlbr. - 7: MV \& VJR (MAF-Lich 16773), Rcc.

Lecanora hagenii (Ach.) Ach. - 5: ELL (BCN-Lich 17411, 17413, $17414,17415,17417,17418,17419,17420,17421,17422,17423$, 17424, 17428, 17431, 17432), Jt, Pd, Qc, Sa, Tsp; 6: ELL (BCNLich 17434, 17442, 17444), Jt, Ps; 8: ELL (BCN-Lich 17464, 17476, 17488, 17516, 17526), Am, Cm, Ps, Qp; 13: ELL (BCNLich 17659, 17661, 17669, 17670, 17672), Qf; 14: ELL (BCNLich 17674, 17694) Bh, Mm.

Lecanora horiza (Ach.) Röhl. - 1: ELL (BCN-Lich 17356, 17370,
17357, 17359, 17360, 17362), VA (VAL-Lich 27455), Gh, Ot, Ssp; 4: ELL (BCN-Lich 17385, 17386, 17387, 17389, 17390, 17392 , 17393, 17396), Qi, Qp; 5: VA (VAL-Lich 27483), Jt; 6: VA (VALLich 27504), Jt; 14: EA, ES \& RA (MACB 102001), ELL (BCNLich $17674,17675,17676)$, Mm, Jt.

Lecanora hybocarpa (Tuck.) Brodo - 1: ELL (BCN-Lich 17370 , 17357, 17359, 17362), Gh, Ot; 4: ELL (BCN-Lich 17384, 17385, 17386, 17387, 17388, 17396), Qi, Qp; 5: ELL (BCN-Lich 17411, 17413, 17415, 17416, 17417, 17418, 17420, 17421, 17422, 17424), Jt, Qc, Tsp; 6: ELL (BCN-Lich 17434, 17444), Jt, Ps; 8: ELL (BCN-Lich 17467, 17468, 17473, 17475, 17487, 17497, 17505), Am, Jc, Psp; 10: ELL (BCN-Lich 17576, 17580, 17581, 17582), Qp; 11: ELL (BCN-Lich 17606, 17619, 17621, 17633, 17634), Qf, Qi; 12: ELL (BCN-Lich 17636, 17647), Ps, Qp; 14: ELL (BCN-Lich 17688, 17692), Mm, Js.

Lecanora leptyrodes G. B. F. Nilsson - 8: ELL (BCN-Lich 17475, 17476, 17486, 17488, 17493, 17494, 17496, 17497, 17499, 17502, 17504, 17505, 17506, 17508, 17516, 17517, 17526), Am, Cm, Jc, Psp, Qp; 11: ELL (BCN-Lich 17615, 17616, 17619), Qi.

Lecanora muralis (Schreb.) Rabenh - 2: ARB (MACB 101506), Rrd; 4: JM (P08849L), Rrd; 5: ARB (MACB 101507), EA, ES \& RA (MACB 102312, 102313), ELS (SANT 11629), Rcz; 6: VA (VALLich 27505), Rcz; 8: BM (SALA-L 4644), SFB \& XLL (BCNLich 17722), Rcc; 10: BM (SALA-L 4994), Rcc; 11: MV \& VJR (MAF-Lich 16842), VA (VAL-Lich 27552), Rcz; 14: BM (SALAL 4845), Rcz.

*Lecanora ochroidea (Ach.) Nyl. - 10: JM (P08850L), Rcc.

Lecanora polytropa (Ehrh.) Rabenh. - 10: BM (SALA-L 4995), Rcc.

** $\neq$ Lecanora printzenii Pérez-Ortega, Vivas \& Hafellner - 10: SPO (SP0-1564), L (Umbilicaria sp.)

Lecanora rupicola (L.) Zahlbr. var. rupicola - 12: MV \& VJR (MAFLich 16856), Rcc.

*Lecanora rupicola var. glaucescens (Sw.) Zahlbr. - 8: JM(P08851L), Rcc.

Lecanora saligna (Schrad.) Zahlbr. - 9: SPO (SPO-1611), Mm.

*Lecanora subcarnea (Lilj.) Ach. - 10: MV \& VJR (MAF-Lich 16835), Rcc.

Lecanora sulphurea (Hoffm.) Ach. - 7: SPO (SPO-1607), Rcc; 8: (BCN-Lich 17731), Rcc; 10: MV \& VJR (MAF-Lich 16838), Rcc.

Lecanora symmicta (Ach.) Ach. - 8: ELL (BCN-Lich 17456, 17458, 17461, 17464), Ps; 12: ELL (BCN-Lich 17639, 17640), Ps.

Lecanora varia (Hoffm.) Ach. - 11: SPO (SPO-1559), Mm; 12: MR (MA-Lich 16417), Ps.

Lecanora viridiatra (Stenh.) Nyl. ex Zahlbr. - 10: MV \& VJR (MAFLich 16829), Rcc.

*Lecidea auriculata Th. Fr. -10: MV \& VJR (MAF-Lich 16825), Rcc.

Lecidea fuscoatra (L.) Ach. - 3: VA (VAL-Lich 27457), Rrd; 4: MV \& VJR (MAF-Lich 16753), Rrd; 10: BM (SALA-L 4996), Rcc.

*Lecidea gypsicola Llimona - 1: MV \& VJR (MAF-Lich 16748), Ys.

**Lecidea lactea Flörke ex Schaer. - 10: MV \& VJR (MAF-Lich 16832), Rcc; 12: SPO (SPO-1566), Rcc.

Lecidea lapicida (Ach.) Ach. - 10: MV \& VJR (MAF-Lich 16836), Rcc.

Lecidella carpathica Körb. - 5: SPO (SPO-1560), Rcz.

Lecidella elaeochroma (Ach.) M. Choisy - 1: ELL (BCN-Lich 17370, 17360, 17362), Gh, Ot; 4: ELL (BCN-Lich 17384, 17385, 17386, 17387, 17388, 17389, 17390, 17391, 17392, 17393, 17396, 17402, 17403, 17404, 17406, 17408, 17410), Mm, Qi, Qp; 5: ELL (BCNLich 17415, 17416, 17417, 17418, 17419, 17431), Jt, Mm, Qc, Sa; 6: ELL (BCN-Lich 17442, 17443, 17444), Ps; 8: ELL (BCNLich 17456, 17458, 17464, 17467, 17468, 17473, 17474, 17475, 
$17476,17486,17488,17497,17502,17503,17504,17505,17506$, 17508, 17516, 17517, 17525, 17526), Am, Cm, Jc, Ps, Psp, Qp; 9: EA, ES \& RA (MACB 102181), Mm; 10: ELL (BCN-Lich 17581, 17582, 17584), Jc, Qp; 11: BM (SALA-L 4952), ELL (BCN-Lich 17605, 17607, 17614, 17616, 17619, 17620, 17621, 17622, 17633, 17634), SPO (SPO-1507), Jt, Qf, Qi; 12: ELL (BCN-Lich 17647, 17650), Qp; 13: ELL (BCN-Lich 17657, 17659, 17660, 17661, 17662, 17668, 17669, 17670, 17672), Qf; 14: ELL (BCN-Lich 17675, 17680, 17685, 17686, 17688, 17690, 17693, 17694), Bh, Jc, Js, Mm, Psp.

Lecidella euphorea (Flörke) Hertel - 5: VA (VAL-Lich 27474), Jt; 11: VA (VAL-Lich 27550), Qi; 12: VA (VAL-Lich 27566), Qi.

**Lecidella flavosorediata (Vězda) Hertel \& Leuckert - 8: ELL (BCNLich 17526), Qp; 10: ELL (BCN-Lich 17580, 17581, 17584), Jc, Qp.

Lecidella stigmatea (Ach.) Hertel \& Leuckert-12: MV \& VJR (MAFLich 16859), Rcc; 14: ELL (BCN-Lich 17709, 17712), VA (VALLich 27620), Rcz, Sue.

** $\neq$ Leciographa weissii Körb. - 8: SFB \& XLL (BCN-Lich 17727), L (Pertusaria flavicans)

**Lepraria crassissima (Hue) Lettau - 14: BM (SALA-L 4830), Rcz.

Lepraria incana (L.) Ach. - 8: BM (SALA-L 4655), Sue; 10: BM (SALA-L 4997), Qp.

**Lepraria leuckertiana (Zedda) L. Saag - 11: ELL (BCN-Lich 17624), Qi.

*Lepraria membranacea (Dicks.) Vain. - 7: SPO (SPO-1498), Rcc.

*Lepraria neglecta (Nyl.) Erichsen - 8: ELL (BCN-Lich 17467), Jc; 10: BM (SALA-L 4998), Mu.

*Lepraria nivalis J. R. Laundon - 6: ELL (BCN-Lich 17444), Ps.

*Lepraria rigidula (B. de Lesd.) Tønsberg - 8: ELL (BCN-Lich 17533), Sue.

**Lepraria umbricola Tønsberg - 4: EA, ES \& RA (MACB 102024, 102027), Sue.

Leprocaulon quisquiliare (Leers) M. Choisy (= L. microscopicum (Vill.) Gams.) - 3: SPO (SPO-1606), Rrd; 4: ARB (MACB 101386), JM (P08835L), Rrd.

**Leproplaca chrysodeta (Vain. ex Räsänen.) J. R. Laundon (= Caloplaca chrysodeta (Vain. ex Räsänen) Dombr.) - 14: BM (SALAL 4831), MV \& VJR (MAF-Lich 16869), Rcz.

**Leproplaca xantholyta (Nyl.) Hue - 14: BM (SALA-L 4826), VA (VAL-Lich 27612), Rcz.

Leptochidium albociliatum (Desm.) M. Choisy - 7: ARB (MACB 101274), MV \& VJR (MAF-Lich 16782), SPO (SPO-1519), Rcc, Smu, Sue; 10: ARB (MACB 101275), BM (SALA-L 4999), ELL (BCN-Lich 17601), Qp, Sue.

Leptogium gelatinosum (With.) J. R. Laundon - 13: BM (SALA-L 4887), Qf.

Leptogium lichenoides (L.) Zahlbr. - 5: BM (SALA-L 5035), Rcz, Sue; 13: ELL (BCN-Lich 17663), ELS (SANT 11860), Qi, Qf; 14: BM (SALA-L 4841), ELL (BCN-Lich 17699, 17707), Sue, $\mathrm{Mu}-\mathrm{Sue}$.

**Leptogium pulvinatum (Hoffm.) Otálora - 5: ELS (SANT 11861), Rcz; 7: MV \& VJR (MAF-Lich 16781), Mu; 11: ELS (SANT 11862), Smu.

Leptogium saturninum (Dicks.) Nyl. - 13: BM, (SALA-L 4877), MR (MA-Lich 16419), SM (MA-Lich 16370), VA (VAL-Lich 27589), Qf.

Leptogium teretiusculum (Flörke) Arnold - 13: VA (VAL-Lich 27590), Qf.

¥ Lichenoconium erodens M.S. Christ. \& D. Hawksw. - 9: VA (VALLich 27524), L (Hypocenomyce scalaris). $\neq$ Lichenoconium lecanorae (Jaap) D. Hawksw. - 11: VA (VAL-Lich 27553), L (Parmelina quercina).

₹ Lichenodiplis lecanorae (Vouaux) Dyko \& D. Hawksw. - 1: SPO (SPO-1527), L (Caloplaca haematites); 7: VA (VAL-Lich 27506), $\mathrm{L}$ (Caloplaca cerina).

ғ Lichenostigma alpinum (R. Sant., Alstrup \& D. Hawksw.) Ertz \& Diederich (= Phaeosporobolus alpinus R. Sant., Alstrup \& D. Hawksw.) - 11: SPO (SPO-1511), L (Lecanora carpinea).

¥ Lichenostigma elongatum Nav.-Ros. \& Hafellner-14: ELL (BCNLich 17689), L (Pertusaria paramerae).

₹ Lichenostigma epipolina Nav.-Ros., Calat. \& Hafellner - 5: SPO (SPO-1593), L (Diplotomma epipolium).

** $\neq$ Lichenostigma epirupestre Pérez-Ortega \& Calat. - 4: SPO (SPO1540), L (Pertusaria pertusa var. rupestris).

ғ Lichenostigma maureri Hafellner (anamorfo) - 7: SPO (SP0-1587), $\mathrm{L}$ (Ramalina sp.).

\# Lichenostigma rouxii Nav.-Ros., Calat. \& Hafellner-5: SPO (SPO1428), VA (VAL-Lich 27484), L (Squamarina cartilaginea).

\# Lichenostigma rugosa G. Thor - 8: SFB \& XLL (BCN-Lich 17728), L (Diploschistes muscorum).

ғ Lichenostigma rupicolae Fdez.-Brime \& Nav.-Ros. - 8: SFB \& XLL (BCN-Lich 17725), L (Pertusaria albescens).

Lobaria scrobiculata (Scop.) P. Gaertn. - 10: EA, ES \& RA (MACB 102112), Qp.

** Lobothallia farinosa (Flörke) A. Nordin, Saviç \& Tibell (= Aspicilia farinosa (Flörke) Flagey) - 14: MR (MA-Lich 16432), SM (MA-Lich 16380), Rcz.

Lobothallia radiosa (Hoffm.) Hafellner (= Aspicilia radiosa (Hoffm.) Poelt \& Leuckert) - 5: ARB (MACB 101387), Rcz; 8: SFB \& XLL (BCN-Lich 17722), Rcc; 11: BM (SALA-L 4762), Rcz; 14: BM (SALA-L 4790), MV \& VJR (MAF-Lich 16863), Rcz.

₹ Marchandiomyces aurantiacus (Lasch) Diederich \& Etayo - 11: MV \& VJR (MAF-Lich 16850), L (Physcia sp.).

* $\neq$ Marchandiomyces corallinus (Roberge) Diederich \& D. Hawksw. - 8: BM (SALA-L 4623), L (Physcia leptalea).

Megaspora verrucosa var. mutabilis (Ach.) Nimis \& Cl. Roux - 1: ELL (BCN-Lich 17370, 17361, 17362), Ot; 14: ELL (BCN-Lich 17676, 17686), Mm.

Megaspora verrucosa (Ach.) Hafellner \& V. Wirth var. verrucosa- 1: ELL (BCN-Lich 17369), Mu-Ys; 6: ELL (BCN-Lich 17445), Sue; 13: VA (VAL-Lich 27594), Qf; 14: ELL (BCN-Lich 17695), Sue.

*Melanelia stygia (L.) Essl. - 7: ARB (MACB 101318), Rcc; 10: MV \& VJR (MAF-Lich 16839), Rcc.

*Melanelixia fuliginosa (Fr. ex Duby) 0. Blanco, A. Crespo, Divakar, Essl., D. Hawksw. subsp. fuliginosa - 8: BM (SALA-L 4649), Qp; 11: BM (SALA-L 4950), Qi; 13: BM (SALA-L 4858), Qf; 14: BM (SALA-L 4812), Jt.

*Melanelixia fuliginosa subsp. glabratula (Lamy) J. R. Laundon - 6: ELL (BCN-Lich 17443), Ps; 8: EA, ES \& RA (MACB 102265), Qp.

Melanelixia glabra (Schaer.) O. Blanco, A. Crespo, Divakar, Essl., D. Hawksw. \& Lumbsch - 8: BM (SALA-L 4599), Qp; 13: ARB (MACB 101319), BM (SALA-L 4850), ELS (SANT 11635), VA (VAL-Lich 27591), Qf.

Melanelixia subargentifera (Nyl.) 0. Blanco, A. Crespo, Divakar, Essl., D. Hawksw. \& Lumbsch - 8: BM (SALA-L 4602), ELL (BCN-Lich 17512), Qp; 11: ELL (BCN-Lich 17604, 17617, 17630), Qf, Qi; 12: VA(VAL-Lich 27569), Qi; 13: ARB (MACB 101320), BM (SALA-L 4847), EA, ES \& RA (MACB 102362, 102363, 102364), ELL (BCN-Lich 17653, 17661, 17666), VA (VAL-Lich 27593), Qf.

Melanelixia subaurifera (Nyl.) O. Blanco, A. Crespo, Divakar, Essl., 
D. Hawksw. \& Lumbsch - 5: ELL (BCN-Lich 17430), Sa; 8: BM (SALA-L 4618), ELL (BCN-Lich 17516, 17520), Qp; 11: ELL (BCN-Lich 17605, 17608), Qi; 12: ELL (BCN-Lich 17647), Qp; 14: BM (SALA-L 4813), ELL (BCN-Lich 17694), Bh.

Melanohalea elegantula (Zahlbr.) O. Blanco, A. Crespo, Divakar, Essl., D. Hawksw. \& Lumbsch - 8: BM (SALA-L 4648), ELL (BCNLich 17463,17464, 17469, 17481, 17494, 17514, 17515, 17516, 17517, 17520, 17522, 17524, 17525, 17527, 17529, 17530), Am, Cm, Ps, Qp; 10: BM (SALA-L 5001), MV \& VJR (MAF-Lich 16824), Qp, Rcc; 14: ELL (BCN-Lich 17674, 17675, 17676, 17684, 17685, 17687, 17688, 17689, 17690, 17694), SM (MALich 16384), Bh, Js, Mm, Ps.

Melanohalea exasperata (De Not.) 0. Blanco, A. Crespo, Divakar, Essl., D. Hawksw. \& Lumbsch - 4: ELL (BCN-Lich 17392), Qi; 6: ELL (BCN-Lich 17442, 17444), Ps; 8: BM (SALA-L 4646), EA, ES \& RA (MACB 102266), ELL (BCN-Lich 17459, 17461, 17462, 17463, 17464, 17468, 17476, 17480, 17481, 17483, 17485, 17486, 17487, 17488, 17490, 17493, 17496, 17499, 17505), ELS (SANT 11631, 11631), Am, ,Cm, Jc, Ps, Psp, Qp; 10: BM (SALA-L 5000), Qp; 11: BM (SALA-L 4780), ELL (BCN-Lich 17604, 17606, 17616, 17630, 17632, 17634), VA (VAL-Lich 27554), Qf, Qi; 12: ELL (BCN-Lich 17640, 17647, 17650), VA (VAL-Lich 27568), Ps, Qi, Qp; 13: ARB (MACB 101317), BM (SALA-L 4851), ELL (BCN-Lich 17669), ELS (SANT 11632), VA (VAL-Lich 27592), Qf; 14: BM (SALA-L 4811), ELL (BCNLich 17678, 17681, 17693, 17694), VA (VAL-Lich 27621), Bh, Jc, Js, Psp.

Melanohalea exasperatula (Nyl.) 0. Blanco, A. Crespo, Divakar, Essl., D. Hawksw. \& Lumbsch - 8: BM (SALA-L 4647), ELL (BCNLich 17456, 17457, 17459, 17460, 17462, 17476, 17482, 17490, 17495, 17503, 17504, 17505, 17508, 17512, 17514, 17515, 17519 , 17524, 17525), ELS (SANT 11634), Am, Cm, Ps, Qp; 9: ELL (BCN-Lich 17551), Ps; 10: BM (SALA-L 5004), ELL (BCN-Lich 17569, 17570, 17581, 17582, 17583, 17584), ELS (SANT 11630), Jc, Mm, Ps, Qp, Rcc; 11: ELL (BCN-Lich 17611), Qi; 12: ELL (BCN-Lich 17640, 17647, 17649, 17650), Ps, Qp; 13: BM (SALA-L 4853), ELL (BCN-Lich 17661, 17672), Qf; 14: BM (SALA-L 4823), ELL (BCN-Lich 17694), Bh.

*Melanohalea infumata (Nyl.) 0. Blanco, A. Crespo, Divakar, Essl., D. Hawksw. \& Lumbsch - 8: BM (SALA-L 4654), Qp; 14: BM (SALA-L 4810), Jt.

Micarea denigrata (Fr.) Hedl. - 9: ELL (BCN-Lich 17555), Ps; 10: ELL (BCN-Lich 17565, 17592), Mm, Ps.

**Micarea globulosella (Nyl.) Coppins - 10: ELL (BCN-Lich 17579, 17582), Qp.

* Micarea melaena (Nyl.) Hedl. - 14: ELL (BCN-Lich 17675), Js.

*Micarea misella (Nyl.) Hedl. - 10: ELL (BCN-Lich 17589), Mm.

** $\neq$ Monodictys epilepraria Kukwa \& Diederich - 4: SPO (SPO-1538), L (Lepraria sp.).

Montanelia disjuncta (Erichsen) Divakar, A. Crespo, Wedin \& Essl. (= Melanelia disjuncta (Erichsen) Essl.) - 8: ELL (BCN-Lich 17537), Rcc.

$\neq$ Muellerella erratica (A. Massal.) Hafellner \& V. John - 4: SPO (SPO1515), L (Protoparmelia sp.).

$\neq$ Muellerella lichenicola (Sommerf.) D. Hawksw. - 4: SPO (SPO1594), L (Tephromela atra); 5: VA (VAL-Lich 27471), L (Caloplaca biatorina).

\# Muellerella pygmaea (Körb.) D. Hawksw. - 10: SFB \& XLL (BCNLich 17737), L (Aspicilia sp.); 12: SPO (SPO-1585), L (Lecidea sp.).
** $\neq$ Nesolechia fusca (Triebel \& Rambold) Pérez-Ortega - 10: SPO (SPO-1541), L (Xanthoparmelia sp.).

Ochrolechia microstictoides Räsänen - 2: ELL (BCN-Lich 17371), Mm; 9: ELL (BCN-Lich 17552), Ps; 10: ELL (BCN-Lich 17560, 17563, 17564, 17565, 17566, 17580), Ps, Qp.

Ochrolechia parella (L.) A. Massal - 8: ELS (SANT 11863) Qp.

Ochrolechia turneri (Sm.) Hasselrot - 8: BM (SALA-L 4619), Qp; 9: EA, ES \& RA (MACB 102194), Ps; 10: MR (MA-Lich 16407), Ps; 13: BM (SALA-L 4859), ELL (BCN-Lich 17655, 17662, 17667, 17669), Qf.

\# Opegrapha glaucomaria (Nyl.) Källsten ex Hafellner - 10: SPO (SPO-1613), L (Lecanora rupicola).

*Ophioparma ventosa (L.) Norman - 10: SM (MA-Lich 16355), Rcc.

** Parmelia barrenoae Divakar, M.C. Molina \& A. Crespo - 8: BM (SALA-L 4607), Qp.

*Parmelia omphalodes (L.) Ach. -12: EA, ES \& RA (MACB 102357), Rcc.

Parmelia saxatilis (L.) Ach. - 4: VA (VAL-Lich 27464), Rrd; 7: SPO (SPO-1520), Rcc; 8: BM (SALA-L 4650), EA, ES \& RA (MACB 102264), ELL (BCN-Lich 17534, 17536), Smu, Rcc; 9: VA (VALLich 27525), Ps; 10: BM (SALA-L 5005), EA, ES \& RA (MACB 102106, 102107), ELL (BCN-Lich 17560, 17564, 17570), ELS (SANT 11637), JM (P08852L), MR (MA-Lich 16392), SM (MALich 16339), VA (VAL-Lich 27530), Ps, Qp, Rcc, Smu; 12: VA (VAL-Lich 27571), Rcc.

Parmelia sulcata Taylor - 6: ELL (BCN-Lich 17442), Ps; 8: BM (SALA-L 4610), EA, ES \& RA (MACB 102259, 102271), ELL (BCNLich 17456, 17458, 17459, 17460, 17461, 17462, 17463, 17464, 17478, 17491, 17500, 17511, 17515, 17516, 17518, 17519, 17524, 17528), ELS (SANT 11636), Am, Cm, Ps, Qp; 11: BM (SALA-L 4954), EA, ES \& RA (MACB 102079), ELL (BCN-Lich 17603, 17616), ELS (SANT 11638), VA (VAL-Lich 27555), Qi; 12: ELL (BCN-Lich 17641), VA (VAL-Lich 27572), Qp, Rcc; 13: ELL (BCN-Lich 17671), VA (VAL-Lich 27595), Qf.

**Parmeliella testacea P. M. Jørg. - 14: ELL (BCN-Lich 17679), Jc. Parmelina carporrhizans (Taylor) Hale-11: ELL (BCN-Lich 17602), Qi; 12: VA (VAL-Lich 27570), Rcc.

Parmelina quercina (Willd.) Hale - 11: MV \& VJR (MAF-Lich 16849), VA (VAL-Lich 27556), Qi.

Parmelina tiliacea (Hoffm.) Hale - 2: ARB (MACB 101321), EA, ES \& RA (MACB 102013), Rrd; 4: EA, ES \& RA (MACB 102033), ELL (BCN-Lich 17407), ELS (SANT 11639), MV \& VJR (MAF-Lich 16752), Mm, Rrd; 7: MV \& VJR (MAF-Lich 16780, 16807), SPO (SPO-1497), Rcc, Smu; 8: BM (SALA-L 4611), EA, ES \& RA (MACB 102270), ELL (BCN-Lich 17458, 17464, 17469, 17476, 17479, 17481, 17485, 17490, 17507, 17511, 17513, 17514, 17519, 17520, 17527, 17530), ELS (SANT 11640), Am, Cm, Jc, Ps, Psp, Qp; 10: BM (SALA-L 5006), EA, ES \& RA (MACB 102103), ELL (BCN-Lich 17572, 17581), SFB \& XLL (BCN-Lich 17744), VA (VAL-Lich 27531), Qp, Rcc; 11: MV \& VJR (MAF-Lich 16848), Qi; 12: ELL (BCN-Lich 17644), VA (VAL-Lich 27573), Qi, Qp; 13: EA, ES \& RA (MACB 102371), Qf.

Parmeliopsis ambigua (Wulfen) Nyl. - 2: ELL (BCN-Lich 17376), Mm; 8: ELS (SANT 11641), Ps; 9: BM (SALA-L 5030), EA, ES \& RA(MACB 102183, 102184, 102185), ELL (BCN-Lich 17549, 17554), ELS (SANT 11642), MV \& VJR (MAF-Lich 16815, 16823), SPO (SPO-1598), VA (VAL-Lich 27526), Ps; 10: ELL (BCN-Lich 17563, 17564, 17565), Ps; 12: ELL (BCN-Lich 17635), Ps 
Parmeliopsis hyperopta (Ach.) Vain. - 9: BM (SALA-L 5031), Pp.

*Parvoplaca tiroliensis (Zahlbr.) Arup, Søchting \& Frödén (= Caloplaca tiroliensis Zahlbr.) - 7: MV \& VJR (MAF-Lich 16779), $\mathrm{Mu}$.

Peltigera canina (L.) Willd. - 8: ELL (BCN-Lich 17532), SFB \& XLL (BCN-Lich 17721), Mu-Rcc; 10: ARB (MACB 101322), ELS (SANT 11667), SM (MA-Lich 16345), Sue; 11: MR (MA-Lich 16412), Sue.

Peltigera collina (Ach.) Schrad. - 8: BM (SALA-L 4643), EA, ES \& RA (MACB 102268, 102263), SFB \& XLL (BCN-Lich 17718), $\mathrm{Mu}, \mathrm{Qp}$, Sue; 10: ARB (MACB 101323), SFB \& XLL (BCN-Lich 17745).

Peltigera malacea (Ach.) Funck - 10: ARB (MACB 101324), MR (MA-Lich 16395), SM (MA-Lich 16344), Sue

Peltigera membranacea (Ach.)- Nyl. - 8: ELL (BCN-Lich 17542), Mu.

Peltigera neckeri Hepp ex Müll. Arg. - 4: MV \& VJR (MAF-Lich 16758), Sue; 5: MV \& VJR (MAF-Lich 16764), Sue

Peltigera polydactylon (Neck.) Hoffm. - 10: ARB (MACB 101325), Sue.

Peltigera ponojensis Gyeln. - 6: ARB (MACB 101326), Sue.

Peltigera rufescens (Weiss) Humb. - 4: ARB (MACB 101327), ELL (BCN-Lich 17382), Sue; 5: ARB (MACB 101328), ELS (SANT 11643), VA (VAL-Lich 27486), Rcz, Sue; 10: BM (SALA-L 5007), MR (MA-Lich 16396), SM (MA-Lich 16346), Sue; 14: ARB (MACB 101329), BM (SALA-L 4834), ELL (BCN-Lich 17706), ELS (SANT 11668), Sue

Pertusaria albescens (Huds.) M. Choisy \& Werner - 2: SFB \& XLL (BCN-Lich 17717), Rrd; 8: BM (SALA-L 4620), ELL (BCN-Lich 17523), ELS (SANT 11864), SFB \& XLL (BCN-Lich 17725 , 17729), Qp, Rcc; 12: ELL (BCN-Lich 17637), Ps; 13: BM (SALA-L 4858), EA, ES \& RA (MACB 102373), ELL (BCN-Lich 17664), Qf; 14: BM (SALA-L 4822), MR (MA-Lich 16431), Js.

Pertusaria amara (Ach.) Nyl. - 4: ARB (MACB 101330), Rrd; 8: ELL (BCN-Lich 17467, 17504), Am, Jc.

Pertusaria coccodes (Ach.) Nyl. - 10: ELL (BCN-Lich 17591, 17597), Mm, Ps; 13: BM (SALA-L 4876), Qf.

**Pertusaria excludens Nyl. - 8: SFB \& XLL (BCN-Lich 17723), Rcc.

Pertusaria flavicans Lamy - 8: SFB \& XLL (BCN-Lich 17727), Rcc; 10: SFB \& XLL (BCN-Lich 17740), Rcc.

Pertusaria hemisphaerica (Flörke) Erichsen -13: BM (SALA-L 4857), Qf.

**Pertusaria lactea (L.) Arnold - 7: SPO (SPO-1548), Rcc.

*Pertusaria leioplaca DC. - 6: ELL (BCN-Lich 17438), Jt.

Pertusaria paramerae A. Crespo \& Vězda - 11: BM (SALA-L 4956), Qi; 12: ELS (SANT 11866), Js; 13: BM (SALA-L 4864), Qf; 14: BM (SALA-L 4819), ELL (BCN-Lich 17683, 17684, 17685, 17689, 17690), Jc, Js.

Pertusaria pertusa (L.) Tuck. - 7: MV \& VJR (MAF-Lich 16802), Rcc. Pertusaria pertusa var. rupestris (DC.) Dalla Torre \& Sarnth. - 10: SPO (SPO-1568), L.

**Pertusaria pluripuncta Nyl. - 8: SFB \& XLL (BCN-Lich 17719, 17723, 17727, 17733), Rcc; 10: SFB \& XLL (BCN-Lich 17753), Rcc.

Pertusaria pseudocorallina (Lilj.) Arnold - 4: MV \& VJR (MAF-Lich 16756), SPO (SPO-1536), Rrd; 8: JM (P08853L), SFB \& XLL (BCN-Lich 17723, 17731), Rcc; 10: BM (SALA-L 5008), SFB \& XLL (BCN-Lich 17738), Rcc.

Pertusaria rupicola (Fr.) Harm. - 3: VA (VAL-Lich 27459), Rrd; 4: ARB (MACB 101331), EA, ES \& RA (MACB 102034, 102035, 102036), MV \& VJR (MAF-Lich 16755), SPO (SPO-1603), Rrd;
8: EA, ES \& RA (MACB 102229), Rcc; 10: VA (VAL-Lich 27532), Rcc.

* $\neq$ Phacopsis oxyspora (Tul.) Triebel \& Rambold - 10: VA (VAL-Lich 27533), L (Xanthoparmelia pulla).

*Phaeophyscia cernohorskyi (Nádv.) Essl. - 1: VA (VAL-Lich 27515), Ot.

Phaeophyscia nigricans (Flörke) Moberg - 1: BM (SALA-L 4978), Qp.

Phaeophyscia orbicularis (Neck.) Moberg - 1: BM (SALA-L 4977), ELL (BCN-Lich 17356), Ssp; 5: EA, ES \& RA (MACB 102302), ELL (BCN-Lich 17424, 17428, 17430, 17431, 17432), VA (VALLich 27487), Jt, Pd, Sa, Tsp; 8: ELL (BCN-Lich 17517), SFB \& XLL (BCN-Lich 17722), Qp, Rcc; 11: BM (SALA-L 4784), ELL (BCN-Lich 17614, 17616, 17617, 17618), Qi; 13: BM (SALA-L 4856), ELL (BCN-Lich 17657, 17666, 17670), Qf.

Phlyctis argena (Ach.) Flot. - 4: ELL (BCN-Lich 17388), Qi; 8: ELL (BCN-Lich 17466, 17472), Jc; 11: BM (SALA-L 4962), Qi; 13: ARB (MACB 101332), BM (SALA-L 4889), Qf.

\# Phoma epiphyscia Vouaux. - 13: BM (SALA-L 4888), L (Physcia stellaris).

Physcia adscendens (Fr.) H. Olivier - 1: ELL (BCN-Lich 17356, 17359, 17360), VA (VAL-Lich 27456), Gh, Ot, Ssp; 4: ELL (BCNLich 17385, 17390, 17392, 17393, 17396), MV \& VJR (MAFLich 16757), Qi, Qp, Rrd; 5: ELL (BCN-Lich 17413, 17414, 17416, 17420, 17428, 17429, 17430, 17431, 17432), VA (VALLich 27488, 27494), Jt, Pd, Qc, Sa, Tsp; 6: ELL (BCN-Lich 17437, 17443, 17444), Jt, Ps; 8: BM (SALA-L 4621), ELL (BCN-Lich 17462, 17464, 17476, 17483, 17485, 17488, 17490, 17491, 17494, 17495, 17502, 17504, 17505, 17512, 17529), Am, Cm, Ps, Psp, Qp; 11: BM (SALA-L 4959), EA, ES \& RA (MACB 102054), ELL (BCN-Lich 17604, 17605, 17607, 17612, 17616, 17617, 17619, 17621, 17632, 17633, 17634), VA (VAL-Lich 27550), Qi, Qf; 12: ELL (BCN-Lich 17650), Qp; 13: BM (SALA-L 4852), EA, ES \& RA (MACB 101987), ELL (BCN-Lich 17655, 17657, $17658,17660,17661,17668,17669,17670)$, Of: 14: BM (SALA-L 4824), ELL (BCN-Lich 17679, 17681, 17686, 17687 , 17688, 17694), MR (MA-Lich 16434), Bh, Jc, Js.

Physcia aipolia (Ehrh. ex Humb.) Fürnr. - 5: ARB (MACB 101333), VA (VAL-Lich 27489), Jt; 6: ELL (BCN-Lich 17441), Ps; 8: EA, ES \& RA (MACB 102260), ELL (BCN-Lich 17459, 17462, 17463, 17476, 17480, 17483, 17488, 17496, 17499, 17502, 17525), Am, Cm, Ps, Psp, Qp; 10: ELL (BCN-Lich 17574), Qp; 11: ELL (BCNLich 17608), SPO (SPO-1522), Qi, Qf; 12: VA (VAL-Lich 27575), Qi; 13: ARB (MACB 101334), BM (SALA-L 4854), EA, ES \& RA (MACB 102372, 102374, 101986), ELL (BCN-Lich 17656 , 17670, 17673), ELS (SANT 11644), VA (VAL-Lich 27596), Qf.

Physcia biziana (A. Massal.) Zahlbr. -1: ELL (BCN-Lich 17360), Gh; 4: ELL (BCN-Lich 17386, 17392), Qi, Qp; 5: EA, ES \& RA (MACB 102301), ELL (BCN-Lich 17413, 17414, 17419, 17422, 17423, 17424, 17429, 17431, 17432), Jt, Pd, Sa, Tsp; 6: ELL (BCN-Lich 17433, 17436), Jt; 10: ELL (BCN-Lich 17598), Ps; 11: BM (SALA-L 4779), ELL (BCN-Lich 17615, 17616), Qi; 13: BM (SALA-L 4855), ELL (BCN-Lich 17661), Qf; 14: ELL (BCNLich 17678), Jc.

Physcia caesia (Hoffm.) Hampe ex Fürnr. - 8: BM (SALA-L 4653), SFB \& XLL (BCN-Lich 17731), Rcc; 10: BM (SALA-L 5009), ELL (BCN-Lich 17598), ELS (SANT 11617), Ps, Rcc; 11: BM (SALA-L 4787), Qi.

* Physcia clementei (Turner) Lynge - 2: ELS (SANT 11646), Mm; 4: ELS (SANT 11645), Mm.

*Physcia dimidiata (Arnold) Nyl. - 10: ELS (SANT 11647), Rcc. 
*Physcia dubia (Hoffm.) Lettau - 2: ARB (MACB 101335), EA, ES \& RA (MACB 102017, 102018, 102019), Rrd; 8: BM (SALA-L 4603), Qp; 11: BM (SALA-L 4785), Qi.

Physcia leptalea (Ach.) DC. - 12: VA (VAL-Lich 27576), Qi; 13: BM (SALA-L 4866), Qf; 14: BM (SALA-L 4829), Jt.

Physcia stellaris (L.) Nyl. - 4: ELL (BCN-Lich 17384), Qp; 5: EA, ES \& RA (MACB 102300), Gs; 7: MV \& VJR (MAF-Lich 16799), Mm; 8: BM (SALA-L 4622), Qp; 12: ELL (BCN-Lich 17650), Qp; 13: BM (SALA-L 4859), Qf.

Physcia tenella (Scop.) DC. - 6: VA (VAL-Lich 27513), Jt; 8: ELL (BCN-Lich 17505, 17512), ELS (SANT 11658), Am, Qp; 11: ELL (BCN-Lich 17611), Qi; 13: BM (SALA-L 4871), Qf; 14: ELL (BCN-Lich 17691), Js.

Physconia detersa (Nyl.) Poelt - 7: MV \& VJR (MAF-Lich 16784), Smu; 8: BM (SALA-L 4604), ELL (BCN-Lich 17471, 17472), SFB \& XLL (BCN-Lich 17734), Jc, Pertusaria pluripuncta; 10: BM (SALA-L 5010), Qp; 13: BM (SALA-L 4861), Qf

Physconia distorta (With.) J. R. Laundon - 11: EA, ES \& RA (MACB 102057), Qi; 13: BM (SALA-L 4872), EA, ES \& RA (MACB 101988), VA (VAL-Lich 27597), Qf.

Physconia enteroxantha (Nyl.) Poelt - 7: MV \& VJR (MAF-Lich 16797), SPO (SPO-1534), Rcc, Smu; 8: BM (SALA-L 4606), ELL (BCN-Lich 17466, 17476, 17479, 17481, 17482, 17489, 17490, 17491, 17503, 17504, 17505, 17516, 17520, 17529, 17531), Am, Cm, Jc, Qp; 10: BM (SALA-L 5011), Qp; 11: BM (SALA-L 4953), EA, ES \& RA (MACB 102055, 102056), ELL (BCN-Lich 17609, 17611, 17614, 17617, 17618, 17632), ELS (SANT 11670), MR (MA-Lich 16411), SM (MA-Lich 16358), Qf, Qi; 12: VA (VAL-Lich 27577), Qi; 13: BM (SALA-L 4862), EA, ES \& RA (MACB 101990), ELL (BCN-Lich 17652, 17654, 17655, 17656, $17657,17658,17660,17661,17662,17663,17666,17667,17669$, 17670, 17672), Qf

Physconia grisea (Lam.) Poelt - 8: BM (SALA-L 4642), ELL (BCNLich 17513), Qp; 11: BM (SALA-L 4934), Qi; 13: BM (SALAL 4872), Qf.

*Physconia muscigena (Ach.) Poelt - 2: ARB (MACB 101336), Rrd; 7: ARB (MACB 101337), Rcc; 12: ELL (BCN-Lich 17646), Qp.

Physconia perisidiosa (Erichsen) Moberg - 7: MV \& VJR (MAF-Lich 16783), Smu; 8: BM (SALA-L 4641), EA, ES \& RA (MACB 102261), ELL (BCN-Lich 17482, 17485, 17495), Am, Cm, Psp, Qp; 11: EA, ES \& RA (MACB 102077), Qi; 12: VA (VAL-Lich 27578), Qi; 13: ARB (MACB 101338), BM (SALA-L 4863), ELL (BCN-Lich 17654, 17655, 17658, 17668, 17672), ELS (SANT 11669), Qf; 14: BM (SALA-L 4828), Jt.

Placidium pilosellum (Breuss) Breuss - 1: ARB (MACB 101508), Sue; 5: JM (PO8855L), Rcz; 6: JM (P08855L), Rcz.

**Placidium semaforonense (Breuss) Breuss - 5: ELL (BCN-Lich 17427), Sue; 14: ELL (BCN-Lich 17699, 17701, 17709), Sue.

Placidium squamulosum (Ach.) Breuss -5: ARB (MACB 101509), Rcz.

Placocarpus schaereri (Fr.) Breuss - 11: ARB (MACB 101339), BM (SALA-L 4934), MV \& VJR (MAF-Lich 16847), SPO (SPO1561), VA (VAL-Lich 27557), Rcz.

Placynthiella icmalea (Ach.) Coppins \& P. James - 2: ELL (BCN-Lich 17377), Mm; 9: ELL (BCN-Lich 17557), Mm; 10: ELL (BCNLich 17588, 17590, 17594, 17598), Mm, Ps.

Placynthiella uliginosa (Schrad.) Coppins \& P. James - 14: ELL (BCNLich 17687), Js.

*Placynthium hungaricum Gyeln. - 5: ARB (MACB 101295), Rcz; 14: ARB (MACB 101298), Rcz.

Placynthium nigrum (Huds.) Gray - 5: ARB (MACB 101299), MV \&
VJR (MAF-Lich 16761, 16762), Rcz; 11: ARB (MACB 101301), Rcz; 14: ARB (MACB 101300), BM (SALA-L 4832), MV \& VJR (MAF-Lich 16864), Rcz.

*Placynthium subradiatum (Nyl.) Arnold - 14: ARB (MACB 101295), Rcz.

*Placynthium tremniacum (A. Massal.) Jatta -5: ELS (SANT 11650), Rcz; 14: JM (P08856L), Rcz.

Platismatia glauca (L.) W.L. Culb. \& C.F. Culb. - 9: EA, ES \& RA (MACB 102180), ELS (SANT 11649), MV \& VJR (MAF-Lich 16814), VA (VAL-Lich 27527), Ps; 10: BM (SALA-L 5012), EA, ES \& RA (MACB 102104, 102105), ELL (BCN-Lich 17560 , 17564, 17575), MR (MA-Lich 16406), SM (MA-Lich 16340), VA (VAL-Lich 27534), Ps, Qp, Rcc.

*Pleopsidium flavum Körb. - 7: ARB (MACB 101340), MV \& VJR (MAF-Lich 16787), SPO (SPO-1513), Rcc; 10: BM (SALA-L 4982), EA, ES \& RA (MACB 102108, 102109), ELS (SANT 11686), MV \& VJR (MAF-Lich 16826), SFB \& XLL (BCN-Lich 17742), VA (VAL-Lich 27535), Rcc.

Pleurosticta acetabulum (Neck.) Elix \& Lumbsch - 8: BM (SALA-L 4601, 4646), EA, ES \& RA (MACB 102243, 102244, 102254), ELL (BCN-Lich 17470, 17476, 17484, 17489, 17491, 17492, 17499, 17501, 17510, 17515, 17518, 17523, 17527, 17529), ELS (SANT 11651), JM (P08857L), Am, Cm, Jc, Psp, Qp; 11: BM (SALA-L 4778), EA, ES \& RA (MACB 102080), ELL (BCN-Lich 17602, 17610, 17631), Qf, Qi; 12:VA (VAL-Lich 27579), Qi; 13: ARB (MACB 101341), BM (SALA-L 4848), EA, ES \& RA (MACB 102367, 102368, 102370), ELL (BCN-Lich 17652, 17667, 17673), MR (MA-Lich 16421), MV \& VJR (MAF-Lich 16860), SM (MA-Lich 16372), SPO (SPO-1496), VA (VAL-Lich 27598), Qf; 14: ELL (BCN-Lich 17677), Jc.

Polycauliona phlogina (Ach.) Arup, Frödén \& Søchting (= Caloplaca phlogina (Ach.) Flagey) - 6: ELL (BCN-Lich 17438), Jt.

Polycauliona polycarpa (Hoffm.) Frödén, Arup \& Søchting (= Xanthoria polycarpa (Hoffm.) Rieber) - 1: ELL (BCN-Lich 17360), Gh; 11: BM (SALA-L 4786), Qi; 13: BM (SALA-L 4890), Qf; 14: ELL (BCN-Lich 17679, 17694), Bh, Jc.

*Polychidium muscicola (Sw.) Gray - 7: ARB (MACB 101276), MV \& VJR (MAF-Lich 16774), Rcc, Sue; 10: ARB (MACB 101276), ELL (BCN-Lich 17601), Sue.

*** Pronectria pilosa Etayo \& López Silanes - 13: VA (VAL-Lich 27599), L (Collema furfuraceum)

Protoblastenia calva (Dicks.) Zahlbr. - 6: SPO (SPO-1551), Rcz.

Protoparmelia montagnei (Fr.) Sancho \& A. Crespo-10: MV \& VJR (MAF-Lich 16830), SFB \& XLL (BCN-Lich 17737), Rcc.

**Protoparmelia nitens (Pers.) R. Sant. - 12: SPO (SPO-1542), Rcc.

*Pseudephebe pubescens (L.) M. Choisy - 10: MV \& VJR (MAF-Lich 16841), SPO (SPO-1574), Rcc.

Pseudevernia furfuracea (L.) Zopf - 2: ELL (BCN-Lich 17377), Mm; 4: EA, ES \& RA (MACB 102028), ELL (BCN-Lich 17394, 17398), MV \& VJR (MAF-Lich 16754), Pp, Rrd; 6: ELL (BCN-Lich 17440, 17443), Ps; 7: MV \& VJR (MAF-Lich 16808), Rcc; 8: BM (SALA-L 4612), EA, ES \& RA (MACB 102242), ELL (BCN-Lich 17455, 17456, 17458, 17460, 17461, 17463, 17465, 17468, 17473, 17474, 17477, 17491), ELS (SANT 11652), Am, Cm, Jc, Ps, Qp; 9: EA, ES \& RA (MACB 102172, 102173, 102174), ELL (BCNLich 17544, 17546, 17547, 17549, 17550, 17554, 17555, 17559), JM(P08847L), MV \& VJR (MAF-Lich 16816, 16821), SPO (SPO1609), VA (VAL-Lich 27528), Mm, Ps; 10: BM (SALA-L 5013), EA, ES \& RA (MACB 102102), ELL (BCN-Lich 17560, 17561, 17562, 17563, 17564, 17565, 17566, 17568, 17569, 17573, 17584), 
MR (MA-Lich 16393), SM (MA-Lich 16341), Jc, Ps, Qp; 12: ELL (BCN-Lich 17636, 17637, 17639, 17640, 17642, 17650), Ps, Qp; 14: BM (SALA-L 4840), ELL (BCN-Lich 17686), Mm, Js.

Psora decipiens (Hedw.) Hoffm. - 1: MV \& VJR (MAF-Lich 16749), Ys; 5: ARB (MACB 101343), EA, ES \& RA (MACB 102309), JM (PO8858L), SPO (SPO-1602), Rcz, Sue; 6: ARB (MACB 101342), ELS (SANT 11653), JM (P08859L), VA (VAL-Lich 27508), Rcz, Sue; 11: ARB (MACB 101344), BM (SALA-L 4774), MR (MALich 16410), Sue; 14: BM (SALA-L 4817), EA, ES \& RA (MACB 101997), ELL (BCN-Lich 17701), SM (MA-Lich 16374), VA (VAL-Lich 27622), Sue.

*Psora globifera (Ach.) A. Massal. - 14: JM (P08860L), MR (MALich 16425), SM (MA-Lich 16381), Rcz, Sue.

Psora testacea Hoffm. - 6: ARB (MACB 101347), JM (P08861L), Rcz.

Psora vallesiaca (Schaer.) Timdal - 6: JM (P08862L), Rcz; 14: ARB (MACB 101348), VA (VAL-Lich 27623), Rcz.

Pycnora xanthococca (Sommerf.) Hafellner - 2: ELL (BCN-Lich 17379), Mm

**Pyrenodesmia chalybaea (Fr.) A. Massal. (= Caloplaca chalybaea (Fr.) Müll. Arg.) - 6: VA (VAL-Lich 27498), Rcz.

Pyrrhospora quernea (Dicks.) Körb. - 12: MR (MA-Lich 16418), Ps.

*Ramalina calicaris (L.) Röhl. - 2: ELL (BCN-Lich 17355), Mm; 8: BM (SALA-L 4630), ELL (BCN-Lich 17524), Qp.

Ramalina capitata (Ach.) Nyl. var. capitata- 7: MV \& VJR (MAFLich 16775, 16800), Rcc; 10: BM (SALA-L 5015), EA, ES \& RA (MACB 102092), SFB \& XLL (BCN-Lich 17751), Rcc.

Ramalina capitata var. digitellata (Nyl.) Nimis - 8: EA, ES \& RA (MACB 102222), Rcc; 10: EA, ES \& RA (MACB 102090, 102091, 102071), Rcc; 12: EA, ES \& RA (MACB 102350, 102351), Rcc.

Ramalina capitata var. protecta (H. Magn.) Nimis - 2: ARB (MACB 101510), Rrd; 4: EA, ES \& RA (MACB 102020), Rrd; 8: EA, ES \& RA (MACB 102224, 102225), Rcc; 10: EA, ES \& RA (MACB 102208, 102209, 102210, 102211, 102212), Rcc; 12: EA, ES \& RA (MACB 102347, 102348, 102369, 102349, 102353, 102071, 102072, 102073, 102074), Rcc.

Ramalina capitata var. strepsilis (Ach.) Motyka - 8: EA, ES \& RA (MACB 102223), Rcc

Ramalina carminae R. Arroyo \& E. Seriñá - 8: EA, ES \& RA (MACB 102058, 102059, 102060, 102062, 102063), Rcc; 10: EA, ES \& RA (MACB 102066, 102067, 102068, 102069, 102070), Rcc; 12: EA, ES \& RA (MACB 102075), Rcc.

Ramalina farinacea (L.) Ach. - 8: BM (SALA-L 4627), EA, ES \& RA (MACB 102219), ELL (BCN-Lich 17515), Qp; 10: BM (SALAL 5014), EA, ES \& RA (MACB 102096), ELL (BCN-Lich 17571), SM (MA-Lich 16343), Qp; 11: BM (SALA-L 4781), EA, ES \& RA (MACB 102081, 102082, 102083), ELL (BCN-Lich 17610, 17628), ELS (SANT 11671), MV \& VJR (MAF-Lich 16853), VA (VAL-Lich 27558), Qi; 12: EA, ES \& RA (MACB 102352), ELL (BCN-Lich 17645), VA (VAL-Lich 27580), Rcc, Qp; 13: BM (SALA-L 4867), MR (MA-Lich 16422), Qf.

Ramalina fastigiata (Pers.) Ach. - 8: BM (SALA-L 4626), Qp; 11: BM (SALA-L 4933), Qi.

Ramalina fraxinea (L.) Ach. - 8: BM (SALA-L 4624), EA, ES \& RA (MACB 102213, 102214, 102215, 102216, 102217, 102218, 102226), ELL (BCN-Lich 17463, 17476, 17479, 17488, 17498, 17518) ELS (SANT 11672, 11871), Am, Cm, Ps, Qp; 11: ELL (BCN-Lich 17629), Qi; 12: SM (MA-Lich 16367), Qp; 13: BM (SALA-L 4868), EA, ES \& RA (MACB 102361), ELL (BCN-Lich
17665, 17673), MR (MA-Lich 16423), MV \& VJR (MAF-Lich 16861), VA (VAL-Lich 27600), Qf.

*Ramalina obtusata (Arnold) Bitter - 9: EA, ES \& RA (MACB 102171), Ps.

Ramalina pollinaria (Westr.) Ach. - 2: EA, ES \& RA (MACB 102009), Rrd; 4: ARB (MACB 101511), EA, ES \& RA (MACB 102022, 102025), ELS (SANT 11673), Pp, Rrd, Sue; 8: EA, ES \& RA (MACB 102220, 102221, 102061, 102064), Rcc; 10: EA, ES \& RA (MACB 102094, 102095, 102065), Rcc; 11: BM (SALA-L 4958), Qi.

Ramalina polymorpha (Lilj.) Ach. - 4: VA (VAL-Lich 27463), Rrd; 10: BM (SALA-L 4516), EA, ES \& RA (MACB 102093, 102097, 102098), VA (VAL-Lich 27536), Rcc; 12: VA (VAL-Lich 27581), Rcc.

Rhizocarpon disporum (Nägeli ex Hepp) Müll. Arg. - 8: JM (P08863L), Rcc.

\# Rhizocarpon epispilum (Nyl.) Zahlbr. - 4: SPO (SPO-1528), L (Pertusaria pseudocorallina); 8: SFB \& XLL (BCN-Lich 17727), L (Pertusaria flavicans); 10: SFB \& XLL (BCN-Lich 17753), VA (VAL-Lich 27532), L (Pertusaria pluripuncta, Pertusaria rupicola).

*Rhizocarpon geminatum Körb. - 8: SFB \& XLL (BCN-Lich 17722, 17724), Rcc; 10: SFB \& XLL (BCN-Lich 17752), Rcc.

Rhizocarpon geographicum (L.) DC. - 8: SFB \& XLL (BCN-Lich 17724), Rcc; 10: BM (SALA-L 5017), SFB \& XLL (BCN-Lich 17740), Rcc; 12: EA, ES \& RA (MACB 102356), Rcc.

*Rhizocarpon simillimum (Anzi) Lettau-12: SPO (SPO-1569), Rcc.

*Rhizoplaca chrysoleuca (Sm.) Zopf - 7: MV \& VJR (MAF-Lich 16777, 16801), SPO (SPO-1502), Rcc; 8: JM (P08864L), Rcc.

*Rhizoplaca melanophthalma (DC.) Leuckert - 7: MV \& VJR (MAFLich 16778, 16805), SPO (SPO-1501), Rcc; 8: JM (P08865L), Rcc.

$\neq$ Rimularia insularis (Nyl.) Rambold \& Hertel - 10: SPO (SPO-1613), L (Lecanora rupicola); 12: MV \& VJR (MAF-Lich 16857), L (Lecanora rupicola).

Rinodina bischoffii (Hepp) A. Massal. - 5: ELS (SANT 11674), Rcz; 6: ELL (BCN-Lich 17453), Rcz

Rinodina capensis Hampe - 10: ELL (BCN-Lich 17577, 17580), Qp.

Rinodina colobina (Ach.) Th. Fr. - 5: ELL (BCN-Lich 17416), Qc; 6: ELL (BCN-Lich 17433), Jt; 11: ELL (BCN-Lich 17621), Qi; 13: ELL (BCN-Lich 17659, 17669, 17670), Qf.

*Rinodina conradii Körb. - 14: ELL (BCN-Lich 17689), Js.

Rinodina llimonae Giralt \& Etayo - 14: ELL (BCN-Lich 17674, 17675, 17687, 17688), Js, Mm.

Rinodina oleae Bagl. - 4: ELL (BCN-Lich 17402, 17403, 17404, 17408), Mm; 5: ELL (BCN-Lich 17421, 17422), Jt; 8: ELL (BCNLich 17506), Am; 11: ELL (BCN-Lich 17613), Qi; 13: ELL (BCN-Lich 17668, 17670, 17672), Qf; 14: ELL (BCN-Lich 17679, 17680, 17685), Jc, Js.

Rinodina plana H. Magn. - 4: ELL (BCN-Lich 17397), Pp; 12: ELL (BCN-Lich 17649), Qp.

Rinodina pyrina (Ach.) Arnold - 1: ELL (BCN-Lich 17356, 17357, 17359, 17360), Gh, Ot, Ssp; 4: ELL (BCN-Lich 17385, 17386, 17387, 17389, 17390, 17392, 17393), Qi, Qp; 5: ELL (BCNLich 17413, 17414, 17415, 17418, 17419, 17420, 17421, 17422, 17424, 17428, 17430, 17431), Jt, Mm, Pd, Qc, Sa, Tsp; 6: ELL (BCN-Lich 17434, 17437, 17444), Jt, Ps; 14: ELL (BCN-Lich 17694), Bh.

Rinodina septentrionalis Malme - 8: ELL (BCN-Lich 17456, 17464, 17476), Cm, Ps; 9: ELL (BCN-Lich 17556), Mm; 10: ELL (BCNLich 17569, 17584, 17590), Jc, Mm, Ps. 
Rinodina sophodes (Ach.) A. Massal. - 8: BM (SALA-L 4638), Qp; 13: BM (SALA-L 4869), Qf.

**Rinodinella dubyanoides (Hepp) H. Mayrhofer \& Poelt - 1: ELS (SANT 11865), Ys; 14: ELL (BCN-Lich 17709, 17711, 17712), Rcz.

Romjularia lurida (Ach.) Timdal - 5: ARB (MACB 101345), Sue; 11: ARB (MACB 101346), Sue.

* $\neq$ Rosellinula haplospora (Th. Fr. \& Almq.) R. Sant. - 12: SPO (SPO1586), L (Aspicilia sp.).

Rusavskia elegans (Link) S. Y. Kondr. \& Kärnefelt (= Xanthoria elegans (Link) Th. Fr.) - 5: ARB (MACB 101362), JM (P08879L), Rcz; 8: JM (P08879L), Rcc; 11: BM (SALA-L 4939), MV \& VJR (MAF-Lich 16845), Rcz.

Sarcogyne regularis Körb. - 5: ELS (SANT 11655), Rcz; 6: SPO (SPO1545), Rcz; 11: ARB (MACB 101349), Rcz.

Schaereria fuscocinerea (Nyl.) Clauzade \& Cl. Roux - 10: SPO (SPO1523), Rcz.

\# Skyttea elachistophora (Nyl.) Sherwood \& D. Hawksw. - 4: SPO (SP0-1549), L (Tephromela atra)

Solorina saccata (L.) Ach. - 14: BM (SALA-L 4791), Sue.

* $\neq$ Sphaerellothecium atryneae (Arnold) Cl. Roux \& Triebel - 10: SPO (SP0-1517), L (Lecanora schwartzii).

₹ Sphaerellothecium cladoniae (Alstrup \& Zhurb.) Hafellner-7: SPO (SP0-1518), L (Cladonia sp.).

* $\neq$ Sphaerellothecium reticulatum (Zopf) Etayo - 7: SPO (SPO-1535), L (Rhizoplaca chrysoleuca).

Squamarina cartilaginea (With.) P. James var. cartilaginea - 1: ELS (SANT 11675), Ys; 5: ARB (MACB 101350), EA, ES \& RA (MACB 102303, 102304, 102305), ELS (SANT 11656), JM (P08866L), VA (VAL-Lich 27490), Rcz, Sue; 6: ARB (MACB 101351), MV \& VJR (MAF-Lich 16769), Rcz; 11: EA, ES \& RA (MACB 102050, 102051, 102052), ELS (SANT 11676), SM (MALich 16360), Rcz, Sue; 14: BM (SALA-L 4792), EA, ES \& RA (MACB 101994), ELS (SANT 11677), MR (MA-Lich 16424), Rcz, Sue.

Squamarina cartilaginea var. pseudocrassa (Mattick) D. Hawksw. - 6: VA (VAL-Lich 27509), Sue; 14: VA (VAL-Lich 27624), Sue.

* Squamarina gypsacea (Sm.) Poelt - 14: MV \& VJR (MAF-Lich 16865, 16871), SPO (SPO-1505), Rcz.

Squamarina lentigera (Weber) Poelt - 1: EA, ES \& RA (MACB 102003), ELL (BCN-Lich 17364), ELS (SANT 11678), JM (P08867L), MV \& VJR (MAF-Lich 16747), Ys; 5: ARB (MACB 101352), ELL (BCN-Lich 17425), VA (VAL-Lich 27491), Rcz, Sue; 11: MR (MA-Lich 16413), SM (MA-Lich 16359), Sue, 14: BM (SALA-L 4820), EA, ES \& RA (MACB 101995, 101996), ELL (BCN-Lich 17696), Sue.

**Squamarina stella-petraea Poelt - 4: ELL (BCN-Lich 17410), Mm.

**Squamulea subsoluta (Nyl.) Arup, Søchting \& Frödén (= Caloplaca subsoluta (Nyl.) Zahlbr. - 4: JM (P08836L), Rrd.

\# Stigmidium acetabuli Calat. \& Triebel - 13: SPO (SPO-1539), VA (VAL-Lich 27601), L (Pleurosticta acetabulum).

** $\neq$ Stigmidium cartilagineae Calat. \& Triebel - 5: SPO (SPO-1436), L (Squamarina cartilaginea).

₹ Stigmidium rouxianum Calat. \& Triebel - 14: SPO (SPO-1434), L (Acarospora cervina).

₹ Stigmidium tabacinae (Arnold) Triebel - 6: ELL (BCN-Lich 17446), L (Toninia sedifolia).

**Strangospora moriformis (Ach.) Stein -2: ELL (BCN-Lich 17381), $\mathrm{Mm}$
**Synalissa symphorea (Ach.) Nyl. - 5: JM (P08868L), Rcz.

Tephromela atra (Huds.) Hafellner - 2: ARB (MACB 101353), Rrd; 3: VA (VAL-Lich 27458), Rrd; 11: BM (SALA-L 4951), Qi; 13: BM (SALA-L 4882), Qf.

** $\neq$ Tetramelas pulverulentus (Anzi) A. Nordin \& Tibell - 13: SPO (SPO-1529), L (Physcia aipolia).

Thelenella muscorum (Th. Fr.) Vain. - 2: ELL (BCN-Lich 17371), Mm; 8: SPO (SPO-1544), Mu.

Toninia albilabra (Dufour) H. Olivier - 5: JM (PO8869L), Rcz; 6: ARB (MACB 101354), Sue; 14: JM (PO8870L), Rcz.

Toninia candida (Weber) Th. Fr. - 5: ARB (MACB 101355), EA, ES \& RA (MACB 102308), ELS (SANT 11657), VA (VAL-Lich 27485), Rcz, Sue; 11: BM (SALA-L 4772), Sue; 14: BM (SALAL 4794), EA, ES \& RA (MACB 102000), JM (P08871L), SM (MA-Lich 16379), Rcz, Sue.

*Toninia cinereovirens (Schaer.) A. Massal. - 5: JM (P08872L), Rcz.

Toninia diffracta (A. Massal.) Zahlbr. - 5: JM (P08873L), Rcz; 6: ELS (SANT 11658), Sue.

Toninia opuntioides (Vill.) Timdal - 5: ELS (SANT 11659), Mu; 14: JM (P08838L), MR (MA-Lich 16433), SM (MA-Lich 16377), Rcz, Sue.

Toninia physaroides (Opiz) Zahlbr. - 1: ARB (MACB 101356), Ys.

Toninia sedifolia (Scop.) Timdal - 1: ARB (MACB 101357), BM (SALA-L 4971), ELL (BCN-Lich 17364, 13768), Ys; 5: ARB (MACB 101358), EA, ES \& RA (MACB 102306, 102307), ELL (BCNLich 17425, 17426, 17427), JM (P08874L), MV \& VJR (MAFLich 16767), Rcz, Sue, 6: ELL (BCN-Lich 17446), VA (VAL-Lich 27510), Sue; 11: ARB (MACB 101359), EA, ES \& RA (MACB 102049), Sue; 14: BM (SALA-L 4821), EA, ES \& RA (MACB 101999), ELL (BCN-Lich 17696, 17699, 17705), MR (MA-Lich 16428), SM (MA-Lich 16385), Sue.

*Toninia squalida (Ach.) A. Massal. - 4: JM (P08875L), Rrd.

Toninia taurica (Szatala) Oxner - 14: JM (P08876L), Rcz.

*Toninia tristis subsp. asiae-centralis (H. Magn.) Timdal - 5: JM (P08868L), Rcz.

*Toninia tristis (Th. Fr.) Th. Fr. subsp. tristis- 14: MR (MA-Lich 16435), SM (MA-Lich 16378), Sue.

Toninia tumidula (Sm.) Zahlbr. - 5: ELS (SANT 11660), Rcz; 6: JM (P08877L), Rcz; 14: BM (SALA-L 4793), Sue.

Trapeliopsis flexuosa (Fr.) Coppins \& P. James - 2: ELL (BCN-Lich 17374, 17378), Mm, Pp; 4: ELL (BCN-Lich 17400), Pp; 9: ELL (BCN-Lich 17553, 17555, 17557), SPO (SPO-1600), Mm, Ps; 10: ELL (BCN-Lich 17568, 17588, 17589, 17590), Mm, Ps.

**Trapeliopsis gelatinosa (Flörke) Coppins \& P. James - 10: ELL (BCN-Lich 17601), Sue.

Trapeliopsis granulosa (Hoffm.) Lumbsch -9: SPO (SPO-1595), Mm.

Tuckermanopsis chlorophylla (Willd.) Hale - 9: BM (SALA-L 5032), JM (P08837L), MR, (MA-Lich 16386), SM (MA-Lich 16338), VA (VAL-Lich 27524), Ps.

*Umbilicaria cinerascens (Arnold) Frey - 7: MV \& VJR (MAF-Lich 16803), Rcc.

*Umbilicaria crustulosa (Ach.) Lamy - 8: EA, ES \& RA (MACB 102235), MV \& VJR (MAF-Lich 16809), Rcc; 10: EA, ES \& RA (MACB 102203, 102204), ELS (SANT 11679), MR (MA-Lich 16401), SM (MA-Lich 16351), Rcc.

*Umbilicaria cylindrica (L.) Delise - 7: MV \& VJR (MAF-Lich 16798), SPO (SPO-1516 (MA)), Rcc; 8: EA, ES \& RA (MACB 102233, 102234), ELL (BCN-Lich 17538), Rcc; 10: BM (SALAL 4520), EA, ES \& RA (MACB 102206), ELS (SANT 11680), VA (VAL-Lich 27537), Rcc. 
*Umbilicaria deusta (L.) Baumg. - 10: EA, ES \& RA (MACB 102197), MR (MA-Lich 16402), SM (MA-Lich 16352), Rcc.

Umbilicaria freyi Codogno, Poelt \& Puntillo - 7: MV \& VJR (MAFLich 16791, 16795), Rcc; 10: EA, ES \& RA (MACB 102205, 102207, 102100), Rcc.

**Umbilicaria grisea Hoffm. - 7: MV \& VJR (MAF-Lich 16792), Rcc; 10: BM (SALA-L 4519), ELS (SANT 11681), Rcc.

*Umbilicaria hirsuta (Sw. ex Westr.) Ach. - 7: MV \& VJR (MAFLich 16793), Rcc; 10: BM (SALA-L 4518), VA (VAL-Lich 27538), Rcc; 12: EA, ES \& RA (MACB 102344), ELS (SANT 11682), Rcc.

**Umbilicaria hyperborea (Ach.) Hoffm. - 8: ELL (BCN-Lich 17539), Rcc; 12: SM (MA-Lich 16365), Rcc.

*Umbilicaria nylanderiana (Zahlbr.) H. Magn. - 10: EA, ES \& RA (MACB 102196), Rcc.

*Umbilicaria pallens Poelt - 8: EA, ES \& RA (MACB 102232, 102240), MV \& VJR (MAF-Lich 16810), Rcc.

Umbilicaria polyphylla (L.) Baumg. - 2: EA, ES \& RA (MACB 102011), Rrd; 3: VA (VAL-Lich 27461), Rrd; 4: ARB (MACB 101360), EA, ES \& RA (MACB 102026), MV \& VJR (MAF-Lich 16760), VA (VAL-Lich 27467), Rrd; 7: MV \& VJR (MAF-Lich 16796), Rcc; 8: EA, ES \& RA (MACB 102230, 102231), ELL (BCN-Lich 17541), SFB \& XLL (BCN-Lich 17730), Rcc; 9: EA, ES \& RA (MACB 102169), Rcc; 10: BM (SALA-L 4521), EA, ES \& RA (MACB 102200, 102201, 102202), ELS (SANT 11683), SFB \& XLL (BCN-Lich 17754), VA (VAL-Lich 27539), Rcc; 12: EA, ES \& RA (MACB 102345), Rcc.

Umbilicaria polyrrhiza (L.) Fr. - 10: BM (SALA-L 4522), Rcc.

Umbilicaria torrefacta (Lightf.) Schrad. - 10: SM (MA-Lich 16349), Rcc; 12: MR (MA-Lich 16416), Rcc.

*Umbilicaria vellea (L.) Ach. - 10: ARB (MACB 101361), BM (SALA-L 4523), EA, ES \& RA (MACB 102198, 102199, 102099), SFB \& XLL (BCN-Lich 17750), Rcc.

*Usnea filipendula Stirt. - 8: ELL (BCN-Lich 17521), Qp; 11: ELL (BCN-Lich 17623), Qi.

**Usnea fulvoreagens (Räsänen) Räsänen -11: EA, ES \& RA (MACB 102340), Qi

**Usnea glabrata (Ach.) Vain. - 11: EA, ES \& RA (MACB 102339), Qi.

Usnea glabrescens (Nyl. ex Vain.) Vain. - 8: ELL (BCN-Lich 17530), Qp; 9: ELL (BCN-Lich 17554), Ps; 10: EA, ES \& RA (MACB 102117), ELL (BCN-Lich 17561), Ps, Qp; 12: ELL (BCN-Lich 17638), Ps.

Usnea hirta (L.) Weber ex F. H. Wigg. - 2: EA, ES \& RA (MACB 102010), Pp; 4: EA, ES \& RA (MACB 102021), ELL (BCNLich 17399), Pp; 8: EA, ES \& RA (MACB 102267), ELL (BCNLich 17460), Ps; 9: EA, ES \& RA (MACB 102159, 102160 , 102161, 102162, 102163, 102164, 102165, 102166, 102167), MV \& VJR (MAF-Lich 16822), Ps; 10: EA, ES \& RA (MACB 102116), Qp; 11: EA, ES \& RA (MACB 102045, 102089, 102329, 102330, 102331), Jt, Qi; 12: EA, ES \& RA (MACB 102346), Qp.

**Usnea lapponica Vain. - 8: EA, ES \& RA (MACB 102273, 102274, 102275, 102276, 102277, 102278, 102279, 102280, 102281, 102282), Qp; 10: EA, ES \& RA (MACB 102113, 102114, 102115), Qp; 11: EA, ES \& RA (MACB 102332, 102333, 102334, 102335, 102336), Qi.

*Usnea subfloridana Stirt. - 9: MV \& VJR (MAF-Lich 16819), Ps.

Usnea substerilis Motyka - 8: EA, ES \& RA (MACB 102272), Qp; 11: EA, ES \& RA (MACB 102337, 102338), Qi
Variospora velana (A. Massal.) Arup, Søchting \& Frödén (= Caloplaca velana (A. Massal.) Du Rietz) - 8: SFB \& XLL (BCN-Lich 17726), Mu.

**Verrucaria glaucina Ach. - 14: VA (VAL-Lich 27620), Rcz.

*Verrucaria macrostoma Dufour ex DC. - 14: ELL (BCN-Lich 17712), Rcz.

*Verrucaria nigrescens Pers. - 1: BM (SALA-L 4976), Ys; 6: ELL (BCN-Lich 17453, 17454), Rcz; 11: BM (SALA-L 4942, 4943), Rcz; 14: BM (SALA-L 4842), ELL (BCN-Lich 17711, 17712), MR (MA-Lich 16426), SM (MA-Lich 16383), Rcz.

¥ Vouauxiella lichenicola (Linds.) Petr. \& Syd. - 7: SPO (SPO-1590), $\mathrm{L}$ (Lecanora cenisia).

F Vouauxiella verrucosa (Vouaux) Petr. \& Syd. - 5: ELL (BCN-Lich 17416), L (Lecanora hybocarpa).

*Xanthocarpia crenulatella (Nyl.) Frödén, Arup \& Søchting (= Caloplaca crenulatella (Nyl.) H. Olivier) - 6: ELL (BCN-Lich 17454), Rcc.

**Xanthoparmelia camtschadalis (Ach.) Hale - 6: ARB (MACB 101512), VA (VAL-Lich 27511), Sue; 11: ARB (MACB 101513), Sue.

Xanthoparmelia conspersa (Ehrh. ex Ach.) Hale - 4: ELL (BCN-Lich 17402, 17403, 17407, 17410), Mm; 10: BM (SALA-L 4524), MR (MA-Lich 16391), SM (MA-Lich 16356), Rcc.

Xanthoparmelia loxodes (Nyl.) O. Blanco, A. Crespo, Elix, D. Hawksw. \& Lumbsch - 8: SFB \& XLL (BCN-Lich 17732), Rcc; 10: SFB $\&$ XLL (BCN-Lich 17743), Rcc.

*Xanthoparmelia pokornyi (Körb.) O. Blanco, A. Crespo, Elix, D. Hawksw. \& Lumbsch - 6: ELL (BCN-Lich 17448), Sue; 14: ELL (BCN-Lich 17704), Sue.

Xanthoparmelia protomatrae (Gyeln.) Hale - 4: ARB (MACB 101514), Sue; 6: ELL (BCN-Lich 17447), Sue - 12: VA (VALLich 27582), Rcc; 14: ELL (BCN-Lich 17703), Sue.

Xanthoparmelia pulla (Ach.) O. Blanco, A. Crespo, Elix, D. Hawksw. \& Lumbsch - 2: ELL (BCN-Lich 17378), Mm; 4: ELS (SANT 11661), Rrd; 8: SFB \& XLL (BCN-Lich 17724), Rcc; 10: BM (SALA-L 5002), VA (VAL-Lich 27540), Rcc.

Xanthoparmelia ryssolea (Ach.) 0. Blanco, A. Crespo, Elix, D. Hawksw. \& Lumbsch- 6: VA (VAL-Lich 27507), Sue.

**Xanthoparmelia somloensis (Gyeln.) Hale - 6: ARB (MACB 101515), EA, ES \& RA (MACB 102318, 102319) Sue; 12: ELS (SANT 11867), VA (VAL-Lich 27583), Rcc; 14: BM (SALA-L 4833), Sue.

Xanthoparmelia tinctina (Maheu \& A. Gillet) Hale-2: EA, ES \& RA (MACB 102012), Rrd; 3: VA (VAL-Lich 27462), Rrd; 4: ARB (MACB 101516), ELS (SANT 11684), Rrd, Sue; 6: ARB (MACB 101517), Sue; 12: EA, ES \& RA (MACB 102358), VA (VAL-Lich 27584), Qi, Rcc.

Xanthoparmelia verruculifera (Nyl.) O. Blanco, A. Crespo, Elix, D. Hawksw. \& Lumbsch - 4: ELL (BCN-Lich 17402, 17403, 17405, 17406, 17408, 17410), Mm; 8: BM (SALA-L 4652), SFB \& XLL (BCN-Lich 17727), Rcc; 10: BM (SALA-L 5003), Rcc.

Xanthoria calcicola Oxner - 1: BM (SALA-L 4975), Rcc; 8: EA, ES \& RA (MACB 102251, 102252, 102253), JM (P08878L), Rcc.

Xanthoria candelaria (L.) Th. Fr. - 6: ELL (BCN-Lich 17433), Jt; 8: ELL (BCN-Lich 17463), Ps; 12: ELL (BCN-Lich 17650), Qp; 13: EA, ES \& RA (MACB 101989), ELL (BCN-Lich 17669), ELS (SANT 11685), Qf.

Xanthoria parietina (L.) Beltr. - 1: ARB (MACB 101363), BM (SALA-L 4975), ELL (BCN-Lich 17356, 17370, 17357, 17359, 17360, 17361, 17362), MV \& VJR (MAF-Lich 16746), VA (VAL-Lich 27453), Gh, Mm, Ot, Ssp; 4: ELL (BCN-Lich 17385), Qp; 5: ARB 
(MACB 101364), EA, ES \& RA (MACB 102298, 102299), ELL (BCN-Lich 17411, 17413, 17414, 17419, 17420, 17421, 17422, 17423, 17424, 17428, 17429, 17430, 17431), VA(VAL-Lich 27492, 27495), Jt, Pd, Qc, Sa, Tsp; 6: EA, ES \& RA (MACB 102323), ELL (BCN-Lich 17433, 17434, 17435, 17436, 17437, 17438, 17443), VA (VAL-Lich 27512), Jt, Ps, Sue; 8: BM (SALA-L 4608), ELL (BCN-Lich 17476, 17481, 17483, 17503), Am, Cm, Psp; 11: BM (SALA-L 4766), EA, ES \& RA (MACB 102085), ELL (BCN-Lich 17602, 17606, 17611, 17616, 17620, 17633, 17634), VA (VALLich 27559), Qf, Qi; 12: VA (VAL-Lich 27585), Qi; 13: BM (SALA-L 4870), ELL (BCN-Lich 17652), VA (VAL-Lich 27602), Qf; 14: BM (SALA-L 4835), ELL (BCN-Lich 17677, 17679), MR (MA-Lich 16429), SM (MA-Lich 16373), Jc, Ps.
F Xanthoriicola physciae (Kalchbr.) D. Hawksw. - 5: VA (VAL-Lich 27493), L (Xanthoria parietina); 11: BM (SALA-L 4765), SPO (SP0-1430), L (Xanthoria parietina); 12: VA (VAL-Lich 27586), $\mathrm{L}$ (Xanthoria parietina).

Xylographa parallela (Ach.) Fr. - 10: ELL (BCN-Lich 17589, 17592), $\mathrm{Mm}$.

Xylographa vitiligo (Ach.) J. R. Laundon - 9: SPO (SPO-1596), Mm.

\section{AGRADECIMIENTOS}

Trabajo financiado por el Proyecto de Investigación del Ministerio de Educación y Ciencia CGL2007-066734-C03-01/BOS.

\section{BIBLIOGRAFÍA}

Aragón, G.; Martínez, I. \& Burgaz, A. R. 1999. Líquenes epifíticos de la Sierra del Tremedal (GuadalajaraTeruel). Teruel 87: 95-110.

Arup, U.; SøChting, U. \& FröDÉn, P. 2013. A new taxonomy of the family Teloschistaceae. Nordic J. Bot. 31: 16-83.

Asso, I. J. 1779. Sinopsis stirpium indigenarum Aragoniae. Marsella.

Atienza, V.; Fos, S.; Sanz, M. J.; Calatayud, V. \& Barreno, E. 1992. Epiphytic lichens from Iberian Paramerae. I. Javalambre mountains (Teruel, Spain). Studia Geobot. 12: 61-67.

BARRERA, I. 1985. Contribución al estudio de la flora y de la vegetación de la Sierra de Albarracín. Departamento de Botánica. Facultad de Ciencias Biológicas. U.C.M. Madrid. Tesis Doctoral.

Burgaz, A. R.; Martínez, I. \& Aragón, G. 2003-2005. Criptógamas: briófitos y líquenes de Teruel. Teruel 90(1): 159200.

Calatayud, V.; Atienza, V. \& BarReno, E. 1995. Lichenicolous fungi from the Iberian Peninsula and the Canary Islands 1. Mycotaxon 55: 363-382.

Calatayud, V.; Barreno, E. \& Eriksson, O. E. 1997. Nimisiostella lichenicola (Lecanorales inc. sed.), a new lichenicolous fungus from Spain. Systema Ascomycetum 15(1-2): 111-116.

Calatayud, V.; Etayo, J. \& Diederich, P. 2001. Paralethariicola aspiciliae (Ostropales, Odontrotremataceae), a new genus and species of lichenicolous fungi. Lichenologist 33(6): 477-482.

Colmeiro, M. 1867a. Enumeración de las criptógamas de España y Portugal, II. Rev. Progr. Ci. Exact. Fis. Nat. 17: 557590. Madrid.

Colmeiro, M. 1867b. Enumeración de las criptógamas de España y Portugal, II. Rev. Progr. Ci. Exact. Fis. Nat. 18: 127157. Madrid.

Colmeiro, M. 1889. Enumeración y revisión de las plantas de la Península hispano-lusitana e Islas Baleares, 5 (Lichenes: 758-875). Madrid.

Crespo, A.; Barreno, E.; Rico, V. J. \& Bueno, A. G. 1980. Catálogo liquénico del desierto de Calanda (Teruel, España). I Anales Jard. Bot. Madrid 36: 43-55.
Divakar, P. K.; Del-Prado, R.; Lumbsch, H. T.; Wedin, M.; EsSLINGER, T. L.; LEAVITT, S. D. \& CRESPO, A. 2012. Diversification of the newly recognized lichen-forming fungal lineage Montanelia (Parmeliaceae, Ascomycota) and its relation to key geological and climatic events. Am. J. Bot. 99(12): 2014-2026.

ErtZ, D.; Lawrey, J. D.; Common, R. S. \& Diederich, P. 2013. Molecular data resolve a new order of Arthoniomycetes sister to the primarily lichenized Arthoniales and composed of black yeasts, lichenicolous and rock-inhabiting species. Fungal Diversity: 1-25.

EтAYo, J. 2010. Líquenes y hongos liquenícolas de Aragón. Guineana 16: 1-501.

Fos, S. 2000-2002. Estudio de la diversidad liquénica epífita de los sabinares y pinares de la Sierra de Javalambre (sudeste de Teruel). Teruel 88-89: 211-245.

Gueidan, C.; Roux, C. \& LutZoni, F. 2007. Using a multigene phylogenetic analysis to assess generic delineation and character evolution in Verrucariaceae (Verrucariales, Ascomycota). Mycological Research 111: 1145-1168.

KaragöZ, Y.; Aslan, A.; Yaz>C>, K. \& Aptroot, A. 2011. Diplotomma, Lecanora, and Xanthoria lichen species new to Turkey. Mycotaxon 115(5): 115-119.

LÁZARO IBIZA, B. 1898. Notas sobre algunos líquenes de España y Portugal. Actas Soc. Esp. Hist. Nat. 27: 180-186, 200-205.

Loscos, F. 1876-1877. Tratado de Plantas de Aragón. $3^{\text {a ed. del }}$ Semanario Farmacéutico. Madrid. Instituto de Estudios Turolenses, Excma. Diputación Provincial de Teruel. 1986: 471-476.

Martínez, I.; Aragón, G. \& Burgaz, A. R. 2003. Estudio de la flora liquénica epifítica de la Sierra de Gúdar (Teruel). Teruel 88: 99-120.

Mateo Sanz, G. 2009. Flora de la Sierra de Albarracín y su comarca (Teruel). Fundación Oroibérico y Jolube Editor.

Nadyeina, 0.; Lutsak, T.; Blum, 0.; Grakhov, V. \& ScheidegGER, C. 2013. Cetraria steppae Savicz is conspecific with $\mathrm{Ce}$ traria aculeata (Schreb.) Fr. according to morphology, secondary chemistry and ecology. Lichenologist 45(6): 841-856.

Navás, L. 1901. El género Parmelia en España. Bol. Soc. Esp. Hist. Nat. 1: 310-317. 
Navás, L. 1910. Líquenes de Aragón. Bol. Soc. Aragonesa Ci. Nat. 9(5): 131-141.

Pardo SaStrón, J. 1901. Apéndice del catálogo de plantas de Torrecilla de Alcañiz. Anales Soc. Esp. Hist. Nat. 10(2): 211-236.

Pardo Sastrón, J. 1903. Catálogo o enumeración de las plantas de Torrecilla de Alcañiz. Bol. Soc. Arag. Ci. Nat. 2: 289297.

PAU, C. 1903. Mi primera excursión botánica. Bol. Soc. Aragonesa Ci. Nat. 2: 154-158.

Rivas Martínez, S. 1987. Memoria del mapa de series de vegetación de España 1: 400.000. ICONA. Ministerio de Agricultura, Pesca y Alimentación, Madrid.
Segarra, J. G. \& Catalán, P. 2001. Catálogo bibliográfico de líquenes de Aragón. Dto. de Agricultura y Economía Agraria. Univ. de Zaragoza.

Sohrabi, M.; Stenroos, S.; Myllys, L.; Søchting, U.; Ahti, T. \& Hyvönen, J. 2013a. Phylogeny and taxonomy of the 'manna lichens'. Mycol. Progress 12: 231-269.

Sohrabi, M.; Leavitt, S. D.; Rico, V. J.; Halici, M. G.; ShresTHa, G. \& Stenroos, S. 2013b. Teuvoa, a new lichen genus in Megasporaceae (Ascomycota: Pertusariales), including Teuvoa junipericola sp. nov. Lichenologist 45(3): 347-360.

ZEDDA, L. 2000. Lecanora leuckertiana sp. nov. (lichenized Ascomycetes, Lecanorales) from Italy, Greece, Morocco and Spain. Nova Hedwigia 71(1): 107-112. 